\title{
Dynamics and local boundary properties of the dawn-side magnetopause under conditions observed by Equator-S
}

\author{
M. W. Dunlop ${ }^{1}$, A. Balogh ${ }^{1}$, W. Baumjohann ${ }^{2}$, G. Haerendel ${ }^{2}$, K.-H. Fornacon ${ }^{3}$, E. Georgescu ${ }^{2,4}$, R. Nakamura ${ }^{2}$, \\ S. Kokubin ${ }^{5}$ \\ ${ }^{1}$ Space and Atmospheric Physics Group, Imperial College, London SW7 2BZ, UK \\ ${ }^{2}$ Max-Planck-Institute für Extraterrestrische Physik, Garching, Germany \\ ${ }^{3}$ Institut für Geophysik und Meteorologie, TUB, Braunschweig, Germany \\ ${ }^{4}$ Institute of Space Sciences, Bucharest, Romania \\ ${ }^{5}$ Solar Terrestrial Environment Lab., Nagoya University, Toyokawa, Aichi 442, Japan
}

Received: 23 February 1999 / Accepted: 21 April 1999

\begin{abstract}
Magnetic field measurements, taken by the magnetometer experiment (MAM) on board the German Equator-S spacecraft, have been used to identify and categorise 131 crossings of the dawn-side magnetopause at low latitude, providing unusual, long duration coverage of the adjacent magnetospheric regions and near magnetosheath. The crossings occurred on 31 orbits, providing unbiased coverage over the full range of local magnetic shear from 06:00 to 10:40 LT. Apogee extent places the spacecraft in conditions associated with intermediate, rather than low, solar wind dynamic pressure, as it processes into the flank region. The apogee of the spacecraft remains close to the magnetopause for mean solar wind pressure. The occurrence of the magnetopause encounters are summarised and are found to compare well with predicted boundary location, where solar wind conditions are known. Most scale with solar wind pressure. Magnetopause shape is also documented and we find that the magnetopause orientation is consistently sunward of a model boundary and is not accounted for by IMF or local magnetic shear conditions. A number of well-established crossings, particularly those at high magnetic shear, or exhibiting unusually high-pressure states, were observed and have been analysed for their boundary characteristics and some details of their boundary and near magnetosheath properties are discussed. Of particular note are the occurrence of mirror-like signatures in the adjacent magnetosheath during a significant fraction of the encounters and a high number of multiple crossings over a long time period. The latter is facilitated by the spacecraft orbit which is designed to remain in the near magnetosheath for average solar wind pressure. For most encounters, a well-ordered, tangential (draped) magnetosheath field is observed and there is little evidence of large deviations in local boundary orientations. Two passes corresponding to close conjunctions of the Geotail spacecraft are analysed to confirm boundary orientation and motion. These further show
\end{abstract}

Correspondence to: M. W. Dunlop, e-mail: m.dunlop@ic.ac.uk evidence of an anti-sunward moving depression on the magnetopause (which is much smaller at Equator-S). The Tsyganenko model field is used routinely to assist in categorising the crossings and some comparison of models is carried out. We note that typically the T87 model fits the data better than the T89 model during conditions of low to intermediate $K_{P}$ index near the magnetopause and also near the dawn-side tail current sheet in the dawnside region.

Key words. Magnetospheric physics (magnetopause, cusp, and boundary layers; magnetosheath; magnetospheric configuration and dynamics).

\section{Introduction}

The Equator-S spacecraft began operations in December 1997 in an inertial, eccentric, near-equatorial orbit, providing good coverage of the dawn-side magnetopause, with the onboard magnetometer experiment (MAM) recording data from 16 December. Crossings have been identified in the magnetometer data set up to the 5 March, 1998, corresponding to a range of local times (LT) at the magnetopause from 06:00-10:40 LT and at a range of heights above the ecliptic plane from about 3-4.8 $R_{E}$. The set of magnetopause crossings recorded by MAM have recently been briefly reported by Dunlop et al. (1999), who surveyed their occurrence in comparison to observed, ambient solar wind conditions; their boundary properties (local magnetic compression and orientation), and the alignment of the magnetosheath field with respect to the set of magnetopause encounters. Here, we report the details of that study more fully and in terms of the local magnetosheath properties, expanding the results. During the survey, a number of unusual events were found, and we discuss these in more detail, together with the results of 
the boundary analysis. We also explicitly analyse the field geometry near the boundary, in comparison to the global magnetic field models (Tsyganenko and Usmanov, 1987; Tsyganenko, 1989), to reveal a systematic deviation between the measured and model field orientation.

The equatorial dawn-side magnetopause and magnetosheath have not been extensively studied. Of the other spacecraft which have traversed this region, ISEE1 and 2 (Russell and Elphic, 1978) and AMPTE IRM and UKS (Bryant et al., 1985) are noteworthy. Older studies of the boundary region, however, have tended to concentrate on the sub-solar region (e.g. Berchem and Russell, 1982; Russell, 1995; Paschmann et al., 1985, 1986; Phan and Paschmann, 1995), and surveys of magnetopause crossings have tended to omit the dawnside (Paschmann et al., 1986; Phan et al., 1994). The apogee of the Equator-S orbit (at $11.5 R_{E}$ ) was much smaller than that of ISEE or AMPTE (at $20.5 R_{E}$ ) and consequently was well placed to sample the magnetopause and near magnetosheath for long periods as the spacecraft moved through apogee. In relative terms, the AMPTE spacecraft rapidly moved away from the magnetopause region after traversal, whereas any excursions deep into the magnetosheath by Equator-S have to arise from large-scale, inward motion of the magnetopause during times when the spacecraft is moving near apogee. The effect is lessened as the orbit evolves further towards the dawn flank. Even for the early orbits, where apogee lies nearer the subsolar point, the expected apogee distance from the magnetopause remains less than about $3 R_{E}$, even at a solar wind ram pressure of $\sim 10 \mathrm{nPa}$.

There have recently been a number studies to investigate the shape and location of the magnetopause boundary as a geometric surface (using compiled data bases of crossings, described e.g. by, Sibeck et al., 1991 and Petrinec et al., 1991) and these have been reviewed by Fairfield (1995). Magnetopause shape, however, is usually determined in these studies by smooth fitting to the crossing locations, rather than by independently checking both location and orientation. Here we check both position and orientation, for the crossing set, noting that the orientation of the observed boundary and model magnetopause systematically miss-align. A possible implication is that a simple, symmetric magnetopause surface may not be sufficient to fit observed crossings.

In Sect. 2, we initially survey all observed traversals across the magnetopause in terms of the solar wind conditions associated with each. In Sect. 3 we describe a number of selected events to illustrate the features of the data set in some detail. Often, the encounters produced a large number of multiple crossings, as well as extended intervals in the boundary region (a few to several hours on occasions). We identify unusual encounters by virtue of high dynamic pressure or particular magnetosheath characteristics. In Sect. 4, we routinely compare results of boundary normal analysis to expected orientation and to indicate tangential alignment of the magnetic field through the crossings. In addition we note that there is a systematic difference between the data, T87 and T89 magnetic field models near the dawn-side flank (Tsyganenko and Usmanov, 1987; Tsyganenko, 1989).

\section{Survey of crossings: occurrence and location}

A total of 31 orbits each contained at least one clear magnetopause traversal, most exhibiting a high number of multiple crossings. Figure 1 shows the occurrence and locations of all the resulting 130 crossings, representing complete or partial excursions into or out of the magnetosheath, together with the projected spacecraft orbit for all 31 passes. The model magnetopause of Formisano et al. (1979a) is also shown for guidance in Fig. 1, with the bow shock (Formisano, 1979b), as cuts at $Z_{\mathrm{GSE}}=4 R_{E}$. The Equator-S spacecraft moves anticlockwise around the orbit, which is inertial and precesses clockwise in GSE for successive passes, as shown in the figure; later passes therefore correspond to earlier local times. The orbit is equatorial and therefore is inclined in GSE above the $X, Y$ plane. The individual crossings all occur at heights which vary between $\sim 2.9$ $4.8 R_{E}$ and Fig. 1 shows that for a mean magnetopause surface, the spacecraft apogee just exits the magnetosphere, but only down to about 09:00 LT. Magnetopause encounters at lower LT (further into the dawn-side) in general, require increasingly high ram pressure conditions to compress the magnetopause sufficiently, biasing crossing occurrence. Fewer crossings are therefore observed for the later passes, and then are typically limited to apogee.

Table 1 summarises this information together with the basic boundary characteristics of the key crossings. The first few event columns in Table 1 show date and times, radial distance $(\mathrm{rr}), \mathrm{X}, \mathrm{Y}, \mathrm{Z}_{\mathrm{GSE}}$ position and geographic LT, followed by the ratio of observed to model magnetic pressure at the magnetopause, used to give an indication of how locally compressed the magnetosphere is at the time of the crossing. The published magnetospheric activity index $\left(K_{P}\right)$ is also listed and is used in the model field comparison. High $K_{P}$ values relate to higher uncertainty in the model coefficients and therefore can indicate a possibly poor comparison. Only a few events, however, occurred during periods of extreme activity $\left(>K_{P}=5\right)$, while several show intermediate levels. The next two columns show the estimated solar wind dynamic pressure and direction of the IMF at the crossing times, computed from one hour solar wind key parameter data obtained predominantly from the WIND spacecraft (Acuna et al., 1995, Russell 1995b). The IMF values are listed to indicate the presence of: southward or northward direction, alignment along the Parker spiral, radially directed fields, or orientation perpendicular to the dawnside bow shock. The WIND spacecraft lies at $\sim 200 R_{E}$ from the Earth and will not necessarily identify individual, small scale features reaching the dawn-side magnetosheath. It should reliably record average conditions convecting with the solar wind, adequate for the purpose here. To complement the IMF information, 


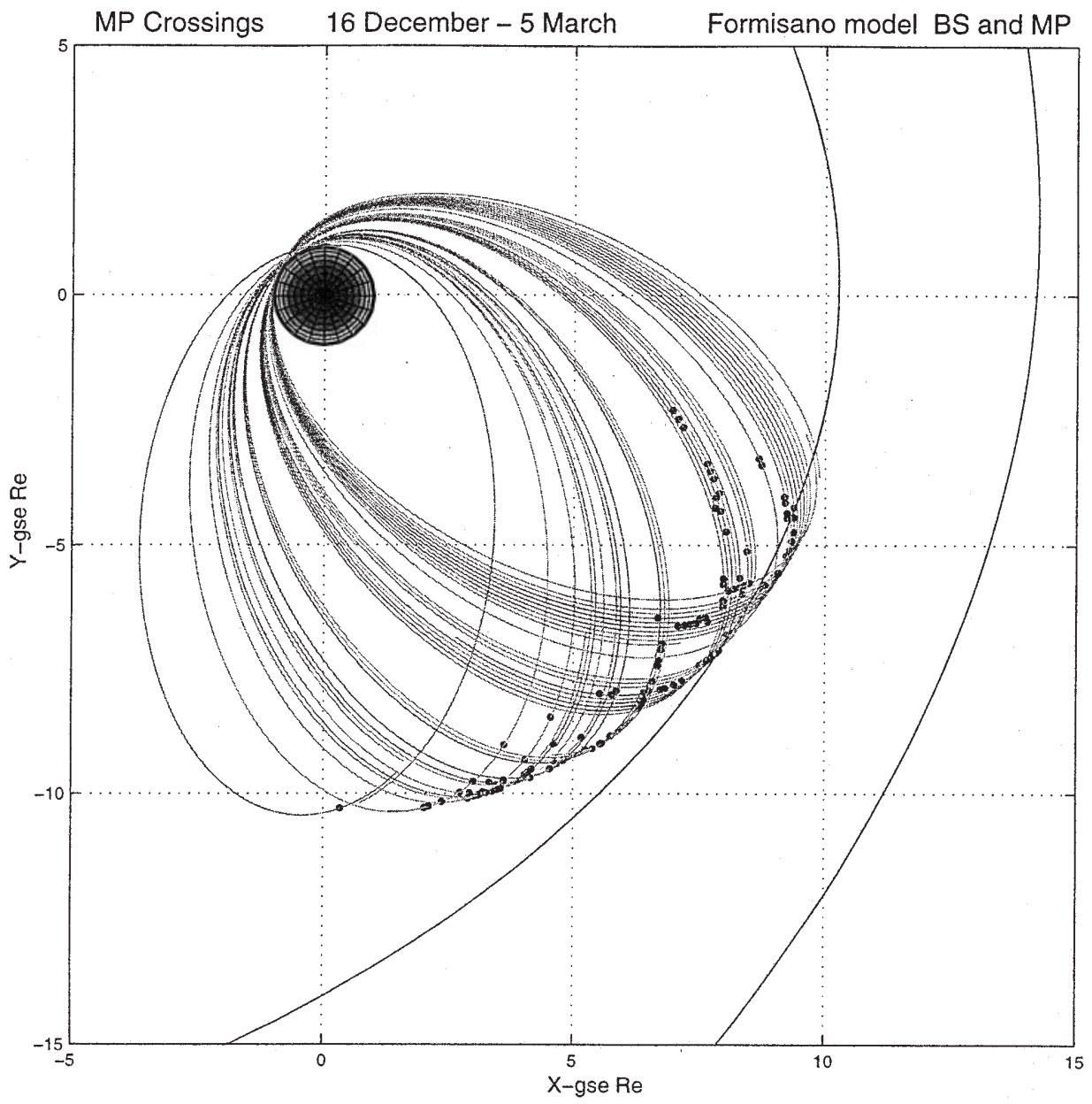

Fig. 1. Overview of the crossing locations. The Equator-S orbit for the 31 passes corresponding to the observations is superimposed to indicate occurrence and cuts through a model bow shock and magnetopause for mean solar wind conditions are also shown (after Dunlop et al., 1999) however, the next column ( $\left.\mathrm{MP}_{\text {shear }}\right)$ shows the local magnetic shear angle between the magnetospheric and magnetosheath fields. This demonstrates that the crossing set covers a range of shear angles, with no obvious biasing to high or low shear at the magnetopause.

With only a couple of exceptions, the majority of the crossings occurred during uncomplicated solar wind conditions; either with no strongly northward or southward IMF and at variable solar wind dynamic pressures, or for low dynamic pressure and strong north/south IMF orientation (see Table 1). This justified comparison to a simple magnetopause surface model (Sibeck et al., 1991) for the pressure sensitive and IMF controlled events separately. This magnetopause model defines the boundary surface through independent sets of pressure sensitive and $B_{Z}^{\mathrm{IMF}}$ dependant coefficients. The column headed $P_{\mathrm{MP}}$ summarises the routine analysis performed on the crossing locations, for both pressure and IMF values. These values in the column correspond to the required solar wind parameters for the best model magnetopause surface to fit each observed crossing position: the required dynamic pressure is written as simple, unsigned values (in $\mathrm{nPa}$ ) and the required $B_{Z}^{\mathrm{IMF}}$ component is indicated by signed values (ranging from -5 to $+5 \mathrm{nT}$ ). Note the close agreement of the pressure values to observed values of $P_{\text {ram }}$ for the majority of the crossings. The values are plotted in the top panel of
Fig. 2, which shows $P_{\text {ram }}$, plotted against the fitted pressures (in $P_{\mathrm{MP}}$ ). This trend supports the view that the majority of events result predominantly from changing solar wind ram pressure, where the IMF showed no clear north/south orientation. The scaling of position with pressure confirms that these crossings are consistent with simple pressure balance response.

Most of the IMF controlled crossings were located closer to the Earth than implied by their $P_{\text {ram }}$ values and corresponded to a range of southward orientations of the IMF. Since only southward IMF conditions produce closer than expected boundary positions for mean pressure values, except far into the flanks, this group uniquely fits the corresponding magnetopause shape and are consistent with the value of $B_{Z}$ taken from the WIND observations. One of the few remaining passes (on the 2 February, at 8:00 LT) closely fitted an extremely northward IMF bin, consistent with its observed value of $B_{Z}$, and in fact was located closer to the Earth than implied by its low observed $P_{\text {ram }}$. The middle panel in Fig. 2 shows a plot of both the pressure values in $P_{\mathrm{MP}}$ and the negative (southward) IMF bins against $\left(B / B_{m}\right)^{2}$. For $P_{\mathrm{MP}}$, the clearly increasing trend supports the view that the majority of events correspond to stable magnetopause position (directly scaling with $P_{\mathrm{MP}}$ ), where the local magnetic compression approximately reflects the ambient measured ram pressure. It is 
Table 1. Properties of main magnetopause crossings

\begin{tabular}{|c|c|c|c|c|c|c|c|c|c|c|c|c|c|}
\hline Date & UT & $\mathrm{rr}$ & $\mathrm{x}$ & $\mathrm{y}$ & $\mathrm{z}$ & LT & $\left(B / B_{m}\right)^{2}$ & $K_{P}$ & $P_{\text {ram }}$ & IMF & $\mathrm{MP}_{\text {shear }}$ & $P_{\mathrm{MP}}$ & $\#$ \\
\hline $18 / 12 / 98$ & 142700 & 10.90 & 8.37 & -5.97 & 3.61 & $9: 38$ & 1.69 & $0+$ & 2.7 & Parker & 90 & 2.3 & 1 \\
\hline $20 / 12 / 97$ & 085300 & 9.72 & 6.68 & -6.46 & 2.86 & 9:03 & 1.96 & 1 & 4.5 & south & 180 & -5 & 1 \\
\hline $21 / 12 / 97$ & 083100 & 10.56 & 7.64 & -6.45 & 3.40 & $9: 19$ & 1.44 & $1-$ & 3.3 & Parker & 170 & 3 & 3 \\
\hline $22 / 12 / 97$ & 063400 & 10.40 & 7.33 & -6.59 & 3.30 & $9: 12$ & 1.96 & $1-$ & 4.2 & radial & 170 & 3.2 & 6 \\
\hline 25/12/97 & 082600 & 11.07 & 9.23 & -4.15 & 4.49 & $10: 23$ & 1.00 & $0+$ & 2.2 & radial & 40 & 2 & 5 \\
\hline 28/12/97 & 050500 & 10.25 & 8.70 & -3.27 & 4.33 & $10: 37$ & 1.44 & 0 & 3.1 & Parker & 30 & 3.3 & 2 \\
\hline $02 / 01 / 98$ & 130600 & 11.41 & 7.76 & -7.22 & 4.23 & 9:08 & 1.00 & 2 & 1.6 & south & 130 & -1 & 1 \\
\hline 03/01/98 & 113600 & 11.42 & 7.67 & -7.32 & 4.24 & 9:07 & 1.00 & $1+$ & 1.3 & south & 160 & -1 & 4 \\
\hline $04 / 01 / 98$ & 103300 & 11.51 & 7.91 & -7.12 & 4.40 & $9: 12$ & 1.56 & $1-$ & 2.0 & south & 80 & -1 & 4 \\
\hline $04 / 01 / 98$ & 141400 & 10.87 & 8.46 & -5.12 & 4.52 & $9: 55$ & 1.20 & $2-$ & 10.8 & south & 30 & -3 & 1 \\
\hline 06/01/98 & 043600 & 10.30 & 5.52 & -7.99 & 3.41 & $8: 35$ & 2.25 & 4 & 3.2 & perp & 130 & 3 & 3 \\
\hline $08 / 01 / 98$ & 110300 & 8.36 & 7.08 & -2.47 & 3.69 & $10: 43$ & 5.00 & $3-$ & 11.5 & south & 80 & 11 & 4 \\
\hline $09 / 01 / 98$ & 073000 & 9.88 & 7.83 & -4.26 & 4.27 & $10: 05$ & 1.98 & $2+$ & 3.1 & south & 70 & -5 & 1 \\
\hline $10 / 01 / 98$ & 033500 & 11.06 & 7.99 & -6.11 & 4.59 & 9:30 & 1.00 & 2 & 2.6 & Parker & 90 & 2.2 & 5 \\
\hline $22 / 01 / 98$ & 235100 & 11.44 & 5.29 & -9.12 & 4.45 & $8: 00$ & 1.00 & 1 & 2.6 & Parker & 170 & 2.6 & 2 \\
\hline $23 / 01 / 98$ & 035900 & 11.02 & 6.67 & -7.43 & 4.66 & $8: 47$ & 1.00 & 1 & 2.6 & Parker & 170 & 2.6 & 10 \\
\hline $24 / 01 / 98$ & 211400 & 11.51 & 5.38 & -9.09 & 4.57 & $8: 02$ & 1.00 & 2 & 2.1 & radial & 120 & 2 & 5 \\
\hline $30 / 01 / 98$ & 143700 & 10.91 & 5.86 & -7.93 & 4.67 & $8: 25$ & 1.00 & $4-$ & 3.3 & south & 180 & 3 & 1 \\
\hline $01 / 02 / 98$ & 054600 & 11.17 & 3.31 & -9.78 & 4.28 & $7: 14$ & 5.37 & $3-$ & 8.2 & radial & 0 & +5 & 2 \\
\hline $01 / 02 / 98$ & 080200 & 11.52 & 4.53 & -9.50 & 4.68 & $7: 41$ & 2.83 & $3-$ & 4.7 & radial & 30 & +5 & 2 \\
\hline $02 / 02 / 98$ & 081200 & 11.30 & 5.16 & -8.86 & 4.76 & $8: 00$ & 1.73 & 1 & 2.0 & north & 30 & +5 & 1 \\
\hline $05 / 02 / 98$ & 221700 & 11.31 & 2.91 & -9.99 & 4.44 & $7: 10$ & 2.83 & 1 & 4.2 & Parker & 40 & 4.2 & 3 \\
\hline $07 / 02 / 98$ & 191500 & 11.46 & 3.12 & -10.02 & 4.61 & 7:09 & 3.00 & 1 & 4.0 & Parker & 100 & 4 & 5 \\
\hline $07 / 02 / 98$ & 212500 & 11.47 & 4.01 & -9.63 & 4.78 & $7: 34$ & 1.73 & 1 & 3.1 & Parker & 110 & 3 & 5 \\
\hline
\end{tabular}

therefore likely that most excursions into the magnetosheath remain close to the magnetopause and any boundary layer.

The high value of $P_{\mathrm{MP}}$ out of trend corresponds to the second encounter on 8 January, which is well fitted by its $P_{\text {ram }}$ value, and therefore primarily controlled by the ambient solar wind pressure. Nevertheless, the encounters occurred during variable IMF conditions so that significant erosion at the magnetopause may partly account for the relatively low apparent magnetic compression. The $B_{Z}^{\mathrm{IMF}}$ values show that all the events controlled by conditions of southward IMF and at low ram pressure have low magnetic compression ratios; also consistent with the presence of erosion. It is predominantly the case, however, that most crossings further into the dawn-side are well (and uniquely) represented by observed, and increasing, dynamic pressure values, as confirmed by the lower panel in Fig. 2, which shows $P_{\mathrm{MP}}$, plotted against LT.

Two passes did correspond to conditions of both medium to high $P_{\text {ram }}$ and strongly southward IMF (20 December and the later crossing on 4 January), but did not scale with pressure. They therefore may correspond to particularly unstable conditions, born out by a contrastingly low value of local magnetic compression. A similar argument can be made for the remaining high pressure pass (1 February), which also did not scale with its observed $P_{\text {ram }}$, but then at $\sim 730 \mathrm{LT}$ and with no clear IMF- $B_{Z}$ direction observed.

The last column in Table 1 shows the number of crossings associated with each encounter listed (note that two, and in one case three, key crossings are indicated for passes containing magnetopause encounters separated by a few hours or more) and many passes show several crossings. This is consistent with the conclusion noted that the spacecraft remained close to the mean magnetopause location, as might be expected from the orbital extent, but is also consistent with large amplitude magnetopause motion. The latter explanation is discounted for the majority of the crossings in Sect. 4.2, where the magnetosheath is discussed in the context of the boundary analysis. Many of the passes show an extended signature containing a variety of distinctive, magnetosheath-like 

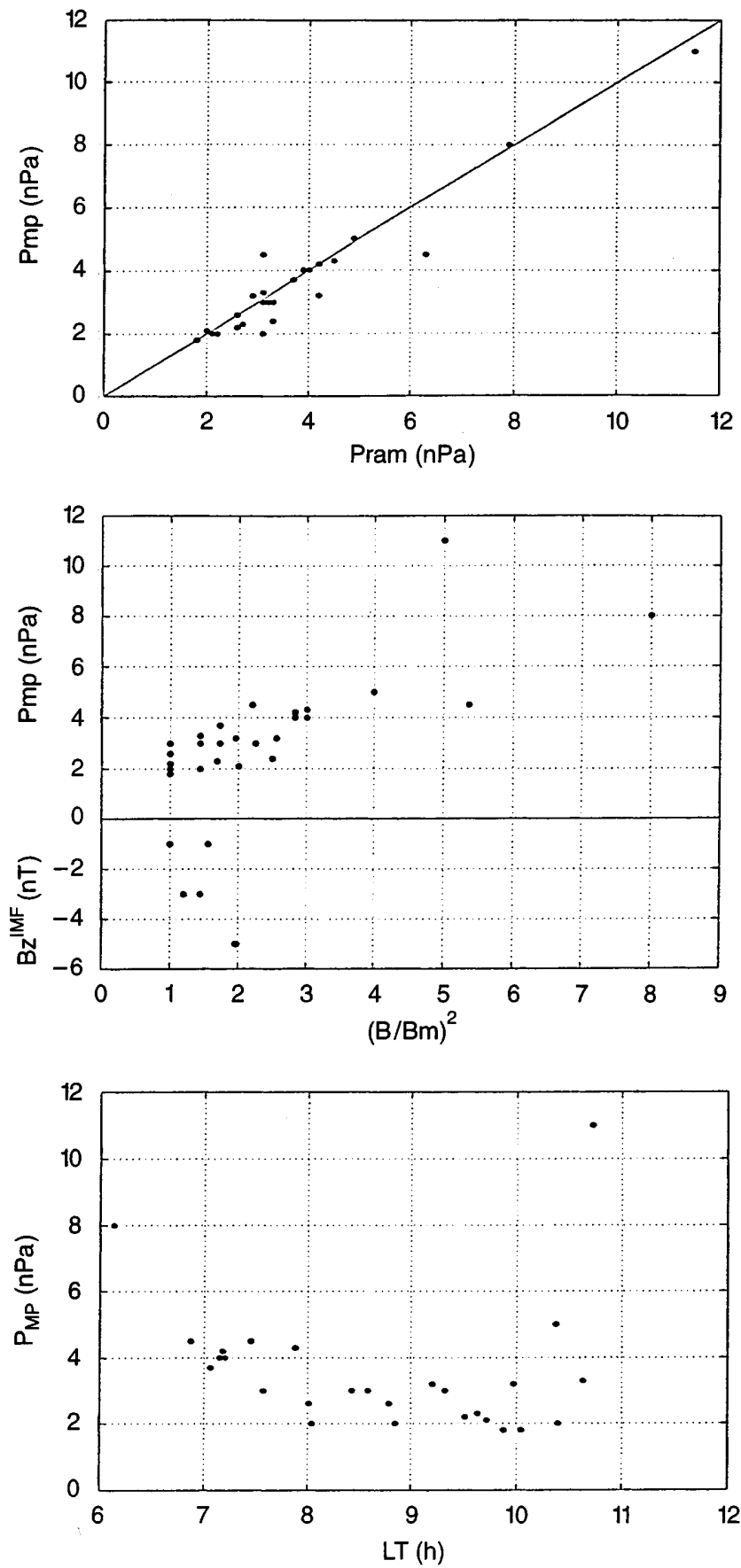

Fig. 2. a Correspondence between the measured $P_{\text {ram }}$ and fitted pressure values for crossings occurring during conditions with no $B_{Z}^{\mathrm{IMF}}$, b correspondence between $\left(B / B_{m}\right)^{2}$ and all crossings fitted to $P_{\mathrm{MP}}$ and $B_{Z}^{\mathrm{IMF}}$, c dependence of $P_{\mathrm{MP}}$ on LT to indicate the biasing of crossing locations to intermediate pressures at low LT

intervals and particular events are described now in Sect. 3 .

\section{Selected event study}

The magnetometer provided high resolution data at $64 \mathrm{~Hz}$ and $128 \mathrm{~Hz}$ resolution. The data employed for this study, however, was predominantly spin averaged data at $\sim 1.5 \mathrm{~s}$ resolution. The data were fully calibrated using routine, inter-comparison of the inboard and outboard sensors and are considered to have an overall uncertainty of between 0.1 and $0.2 \mathrm{nT}$ (Fornacon et al., this issue). The individual crossings are currently identified only by the magnetic field, the only calibrated data set available routinely at the time of this report. Identification is therefore assisted by use of the Tsyganenko model field (see later) which provided a number of comparative features. Firstly, the model trace provides a background configuration for the magnetospheric field which can better reveal the different field orientation in the magnetosheath, at least for high shear events, best identified by sharp changes in field orientation alone. Secondly, it provides a nominal value for the field intensity near the magnetopause with which to compare magnetospheric state.

Typically, in the crossings observed here, the magnetospheric field lies close to the model field orientation and the magnetosphere is often in a magnetically compressed state, with a greater than nominal field magnitude at the magnetopause. Furthermore, most crossings occur for a local magnetosheath field which is directed at a large angle to the magnetospheric field and result in significant magnetic shear across the magnetopause. A problem with identification in the absence of a strong magnetic discontinuity is the risk of confusion with any boundary layer signature. Only a few low shear crossings were observed and have been identified more approximately by changes in field magnitude, or an enhancement of magnetic variance. Discontinuities in the magnetosheath of Heliospheric origin can also usually be discounted (i.e. distinguished from true magnetopause crossings), since they are likely to only rarely correspond to the magnetopause orientation, and hence the model field. Furthermore, the magnetosheath field often exhibited distinctive characteristics, such as mirror mode waves (further analysed in Lucek et al., 1996a, b), which differ from those of the magnetosphere and make miss-identification unlikely.

Below we discuss in some detail the features and character of selected events, summarising the analysis for all magnetopause encounters in the following section.

\subsection{High shear magnetopause: December 16}

Figure $3 \mathrm{a}$ shows an extended interval of the outbound, magnetopause encounter of 16 December shown by Dunlop et al. (submitted to JGR), expressed in GSE polar co-ordinates, where $\theta$ is the latitude of the field and $\phi$ is measured positive from $X_{\mathrm{GSE}}$. The solid traces in each panel are the measured magnetic field data, while the dashed traces show the model magnetic field, computed from the combined IGRF and T87 global magnetospheric field model (Tsyganenko and Usmanov, 1987). Section 4.1 discusses the field model comparison further. The lower panel of Fig. 3a shows the radial distance, from Earth centre, around the spacecraft orbit for the day. The event is chosen to illustrate the identification procedure: it shows very good agreement 

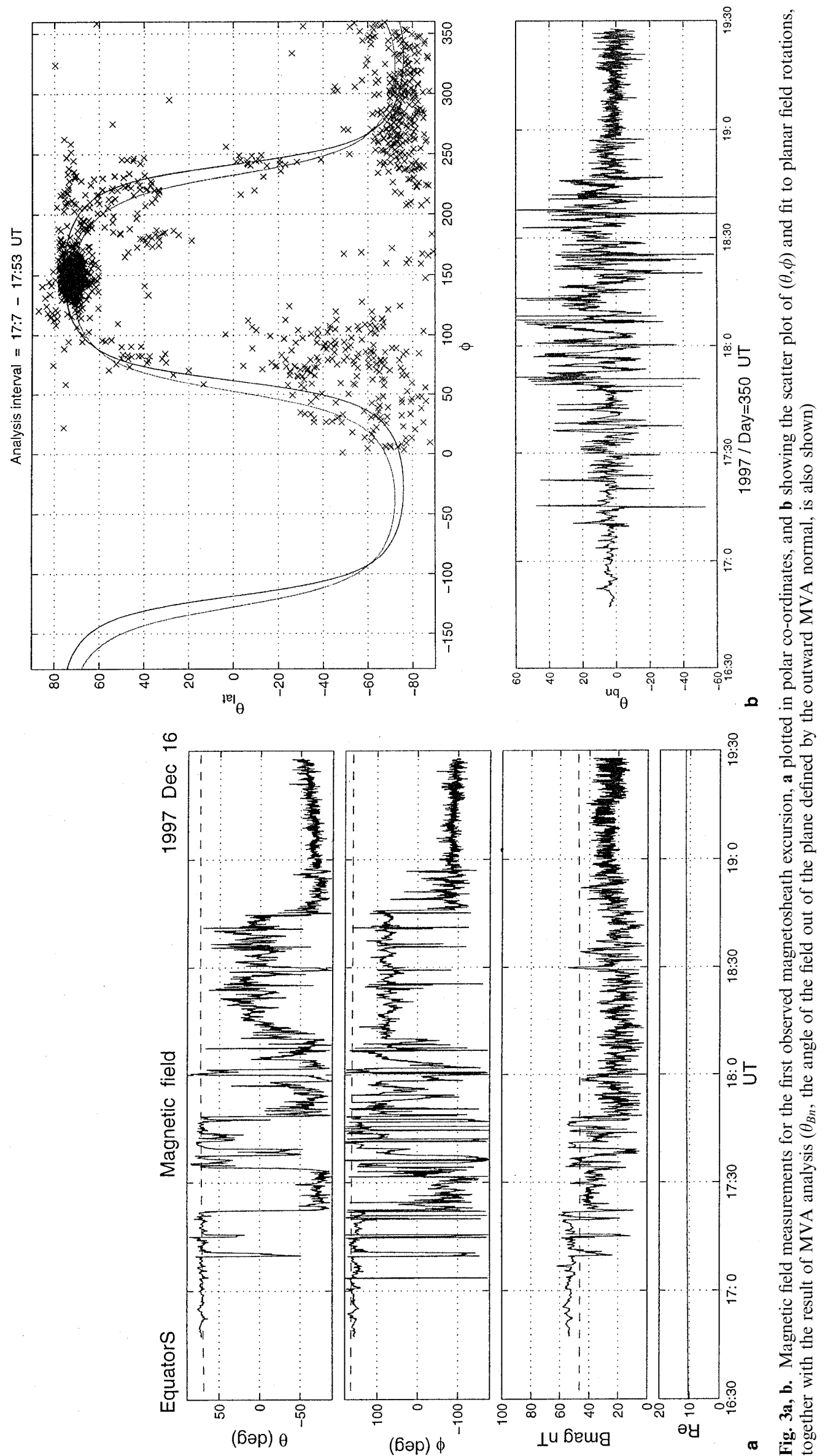
(within a degree) with both the T87 (as shown) and the T89 Tsyganenko models (Tsyganenko, 1989) and clearly corresponds to high magnetic shear conditions between the magnetospheric and magnetosheath fields (corresponding to southward IMF). The basic characteristics of this event are documented in Table 1 , indicating that it occurs at intermediate local time (9:39 LT) and for low magnetospheric activity $\left(K_{P}=1\right)$.

This magnetopause traversal does not correspond to high solar wind ram pressure and the plot here shows that there is little magnetospheric compression near the magnetopause. The magnetopause position was in fact best fitted for the observed southward IMF orientation and the event shows a mixed signature which is fairly typical of magnetopause encounters at high magnetic shear (Russell, 1995). There is a substantial, and abrupt, change in the field magnitude, as well as in the field direction at all crossings at the magnetopause. Although the sheath field does not show large amplitude compressional variations (in $|\mathbf{B}|$ ) initially, beyond 19:00 UT a stable compressional signature does develop which is reminiscent of drift mirror modes (e.g. Anderson and Fuselier, 1993). Although not a long event, there are clearly a large number of individual crossings (as indicated in Table 1), between 17:00 and 18:00 UT, including a number of partial crossings (at 17:09, 17:13, 17:37, 17:41 and 17:47 UT).

The lower panel of Fig. 3b shows the result of minimum variance analysis (Sonnerup and Cahill, 1967) of the key interval containing the crossings (as heads the plot), the mean, minimum variance (MVA) normal lying about $22^{\circ}$ sunward of the model boundary orientation, as indicated in Table 2. In the lower plot, $\theta_{b n}$ is the latitude of the field with respect to the minimum variance direction found for the whole interval. It is clear that, overall, the magnetosheath field lies nearly parallel to the boundary, despite large (up to $\sim 50^{\circ}$ ) fluctuations, predominantly between about 17:45 and 18:45 UT (which corresponds to the different orientation of the magnetosheath field apparent here by the field rotation at 18:45). In this interval, the underlying field direction does turn away slightly from the magnetopause orientation, as might be expected through operation of the slow mode (Southwood and Kivelson, 1992; Song et al., 1992a, b). Beyond this time, however, the field fluctuates around a nearly fixed direction, closely parallel to the boundary. The spectral signature of the compressional variations broadens from a peak at around $10^{-2} \mathrm{~Hz}$, to a shoulder just below $10^{-1} \mathrm{~Hz}$, and reminiscent of the mirror signature. The underlying field magnitude is clearly relatively depressed between this later period and the magnetopause encounters, implying a reduction in field energy (perhaps enhancing the flow) and also suggesting the presence of a slow mode front.

The upper panel of Fig. $3 \mathrm{~b}$ shows a scatter plot of the field orientation for the same interval. Superimposed are two curves: the grey curve represents a fit of the observed magnetic field orientations to variation in a plane $(\mathbf{B} \cdot \mathbf{n}=0)$, while the other represents a variation of the magnetic field vector in the maximum variance plane for the interval, defined by the minimum variance direction (see Farrugia et al., 1991 for a description of the planar analysis). Although closely related through the divergence free nature of the magnetic field, both planes need not be the same, but will closely agree for a 1-D tangential discontinuity. Used in combination with MVA in this way the test provides a visual indication of the nature of the boundary. The scatter plot for this whole interval, in which the field orientations do not remain close to either planar curve, confirms that the individual crossings do not represent TDs. At the same time, the coincidence of the two curves suggests that the minimum variance direction is a good indication of the mean boundary orientation. The result of the boundary analysis is also summarised in Table 2. For the individual crossing intervals the planar and MVA directions deviate from one another. Analysis of the individual crossings suggests that the boundary remains stable with only a few of the crossings showing evidence of small, significant tilts $\left(\sim 10-15^{\circ}\right)$ to the mean normal indicated in Table 2. This small tilting of the boundary, however, is primarily a change in the $(Z, Y)_{\mathrm{GSE}}$ plane and not in $(X, Y)_{\mathrm{GSE}}$. The tilting is consistent with a slight flattening of the magnetopause, with the normal rotating first into $Z$ and then into $Y$, occurring within the re-entry into the magnetosphere (17:34-17:49 UT). The dominant component remains along $X_{\mathrm{GSE}}$. A similar effect is observed during the 11 February traversal, discussed later.

It is inappropriate here to attempt a categorisation of any boundary layer, and associated structure, using the magnetic field data alone, but we note that the magnetosheath interval between the inbound and outbound crossings at 17:22 and 17:34 UT is likely to be closest to the magnetopause. It has the lowest amplitude variations in field magnitude; apart from a decrease in $|\mathbf{B}|$ just outside the magnetopause. The very close agreement of the magnetospheric field orientation to the Tsyganenko model, even at the boundary, is partly consistent with the low value of $K_{P}=1$, but is by no means typical of the events located further into the dawn-side. It more directly shows that there is little magnetic compression above the model field and perhaps results from a high degree of magnetospheric erosion.

\subsection{Ordered magnetosheath, low shear: January 9 and February 1}

In comparison to the event discussed previously, the inbound crossing shown in Fig. 4 represents a location at a similar local time (10:00 LT), but with longer coverage of the magnetosheath. The model field again shows very close agreement for both T87 and T89 (T87 is shown in Fig. 4a) although magnetospheric activity $\left(K_{P}=2+\right)$ and local magnetic compression are a little higher. There appears to be only one magnetopause crossing, which is unfortunately confused by a data gap. It is clear, however, that the magnetosheath field initially lies at low magnetic shear to the magnetospheric field, before turning southward at about 05:00 UT, prior to the magnetopause crossing. The solar wind at the time of the crossing, and the fit to location are also 

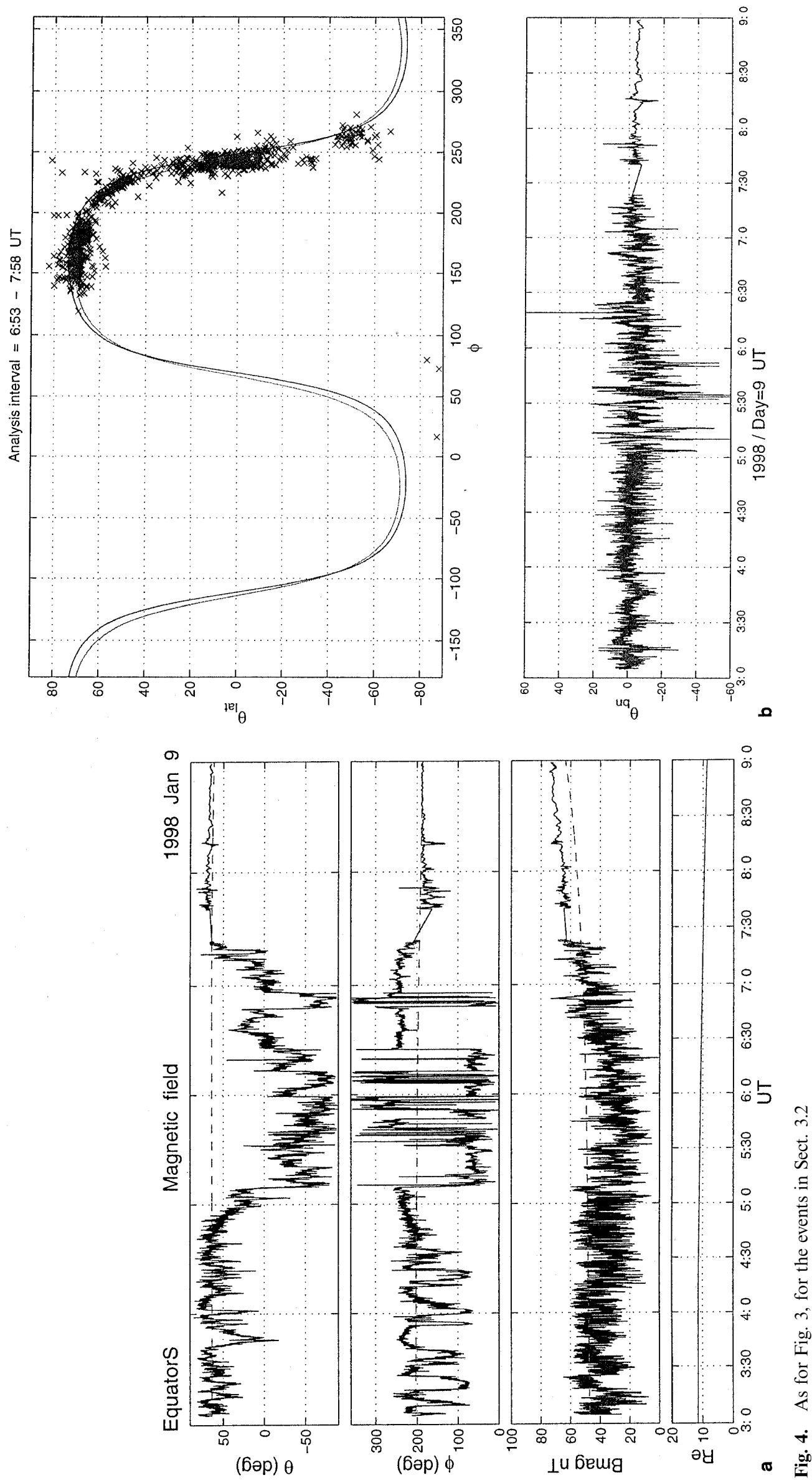
representative of southward orientation of the IMF (as indicated in Table 1). The solar wind ram pressure is estimated at $\sim 3 \mathrm{nPa}$, a similar value to that for the 16 December event. Nevertheless, there is no sharp field rotation at the magnetopause and Fig. 4b shows that the magnetosheath field remains well ordered by the implied MVA normal (calculated over the interval indicated) throughout the observed magnetosheath.

This MVA normal is indicated in Table 2 and although still sunward of the model boundary orientation, it lies within $\sim 10^{\circ}$ of this. Fluctuations in the field direction predominantly remain within $\pm 20^{\circ}$ of the maximum variance plane for the interval, only rarely exceeding this. The spectral signature of the compressional variations (which in this case dominate) broadens to higher frequencies further away from the boundary in a similar way to that seen in the 16 December event (although now the pass is inbound); the character changing just after 05:00 UT. Note, again, the slight depression in field magnitude, after this time, in the region adjacent to the magnetopause. The coincidence of the planar and MVA curves and the well-ordered scatter in Fig. 4b suggest that the variation of the field across the magnetopause is tangential.

The magnetopause traversal on 1 February, shown in Fig. 5, represented a locally low shear event, in which the magnetosheath field remained northward for the majority of the interval until 7:15 UT, after which a number of rotations in magnetosheath field appear; reflecting the fact that the upstream IMF did not have a clear north/ south orientation (see Table 1). The crossings occur at local times further into the dawn-side, however, ranging from 07:14-07:41 LT. In fact, the model field corresponds closely to the measured field only for the T87 model (as shown in Fig. 5a), a result which we discuss further in Sect. 4. For this event magnetospheric activity, local compression of the magnetosphere and solar wind pressure are all high. Although for a much longer interval than the previous events, for the majority of the interval the magnetosheath field for this outbound event is again very closely aligned to the magnetopause orientation implied by MVA, as shown in the lower panel of Fig. 5b. The pair of crossings around 06:00 UT and at $\sim 720$ UT are consistent with a TD at the boundary and show scatter plots which are very similar to that in Fig. $4 \mathrm{~b}$ for 9 January. Both the fitted and MVA planes coincide for all crossings, as illustrated in Fig. $5 \mathrm{~b}$ for the crossing at 06:12 UT (then for very low magnetic field rotation). This analysis of the individual crossings show orientations which agree closely $\left(\sim 5-10^{\circ}\right)$ to the mean normal quoted in Table 2, but the small changes in orientation are significant.

For the event of 1 February, large amplitude, compressional signatures appear adjacent to the magnetopause crossing, although there appears to be an extended period of increased variance inside the magnetopause, typical of the dawn-side events. After 07:15 UT there is an apparent drift in the background magnetosheath field direction by more than $20^{\circ}$ relative to $\theta_{B n}$, away from the implied magnetopause orientation (see the lower panel of Fig. 5b), although it returns to an alignment closer to $\theta_{B n}=0$ at around 8:30 UT. The direction is consistent with that expected to link field lines through the bow shock, in this case for a radial IMF (e.g. Spreiter et al., 1966). This suggests that for this high pressure event the spacecraft does sample the magnetosheath further from the magnetopause than indicated by most of the crossing events discussed here. This is consistent with the view that the crossing location is not stable, arising from the fact that this event did not fit well to the model boundary (Sect. 2). The implication would be that the event moves substantially inwards, away from the spacecraft, after the observed crossings. This is not unusual for the magnetopause, but appears to occur for only a minority of the Equator-S magnetopause encounters.

\subsection{High pressure events: January 8 and March 5}

The two innermost crossings with respect to the Earth (see Fig. 1), for which the solar wind pressure was highest (8 January and 5 March) are of particular interest and are plotted in Fig. 6 and 7. The magnetopause traversal on the 8 January (Fig. 6) has two main encounters, occurring on the inbound leg of the orbit, and therefore corresponds to local times (09:58 and 10:43 UT) nearest the sub-solar magnetopause, and away from the dawn-side. The magnetopause appears to move inwards at the first encounter on this day so that the spacecraft exits into the magnetosheath during only intermediate solar wind conditions, whereas by the second encounter (as indicated in Table 1), it re-enters the magnetosphere at a magnetopause distance which corresponds to a solar wind dynamic pressure of $\sim 11$ $\mathrm{nPa}$. The WIND data indicates that there is a turning of the IMF from north to south, however, between the two encounters on the 8 January. By contrast, the magnetopause traversal on 5 March (Fig. 7) exits the magnetosphere only briefly, at apogee, but at the minimum local time (06:07 UT), farthest into the dawn-side. The rapid increase in the magnetic field intensity just before the main crossing (at 21:58 UT) suggest a rapid and sudden inward motion of the magnetopause. Both events exhibited high magnetic compression at the magnetopause, at high and low magnetic shear, respectively, and for both the magnetopause locations agree well with observed $P_{\text {ram }}$ (which confirm the implied rapid increase in pressure during the 8 January event). Although separated by $\sim 5: 00 \mathrm{~h}$ of LT, both show intense, short intervals of very large amplitude compressional signatures (identified as possible mirror signatures), immediately next to the magnetopause crossing. The times of the main crossings for both are associated with density spikes in the WIND data.

Magnetospheric activity is not dissimilar between the two events $\left(K_{P}=3+\right.$ for the second encounter on 8 January and $K_{P}=4-$ for 5 March), although the first encounter on 8 January occurs during a lower level $\left(K_{P}=2\right)$. The model field is not expected to closely follow the measured field at such high pressure conditions, particularly when activity levels and local mag- 

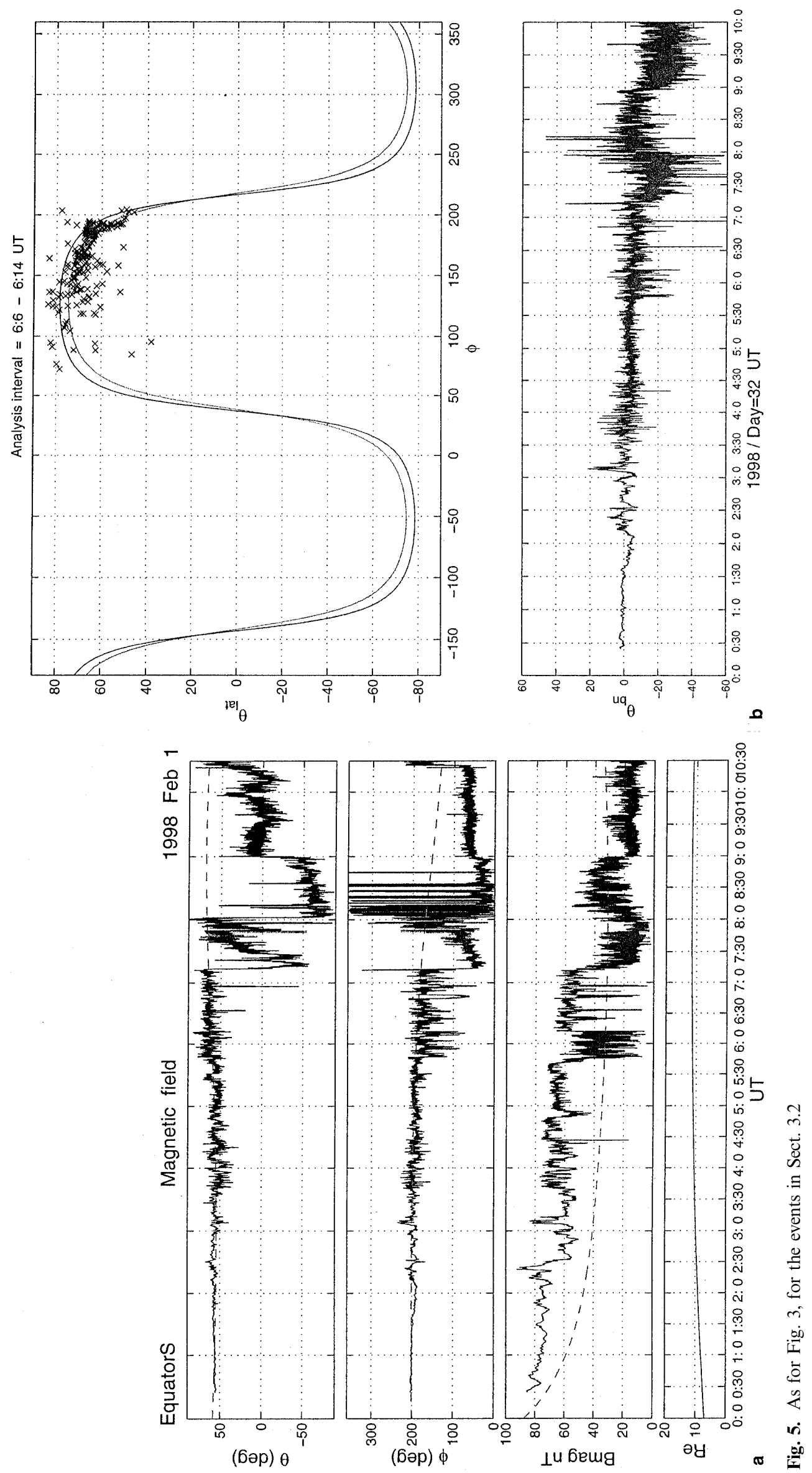

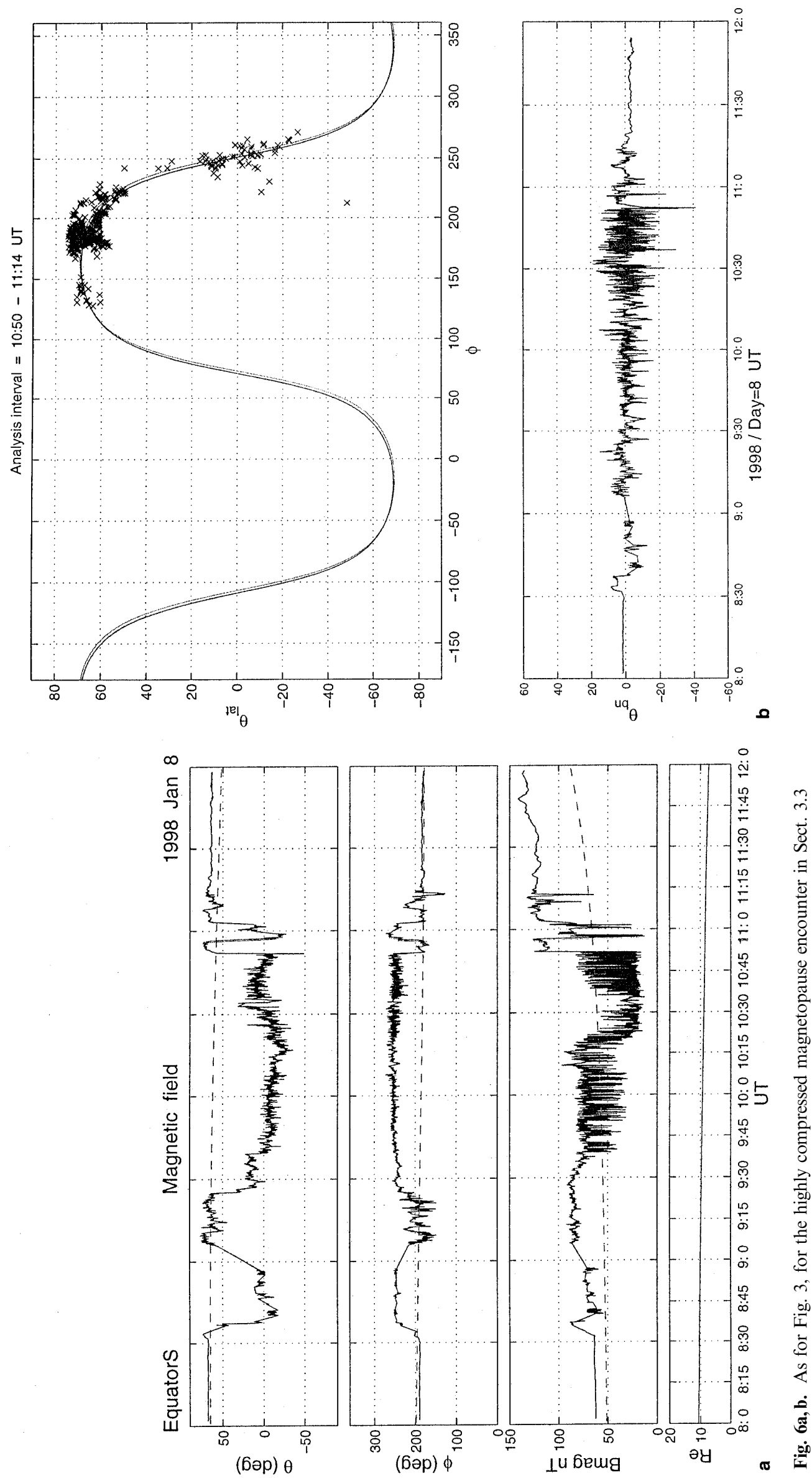


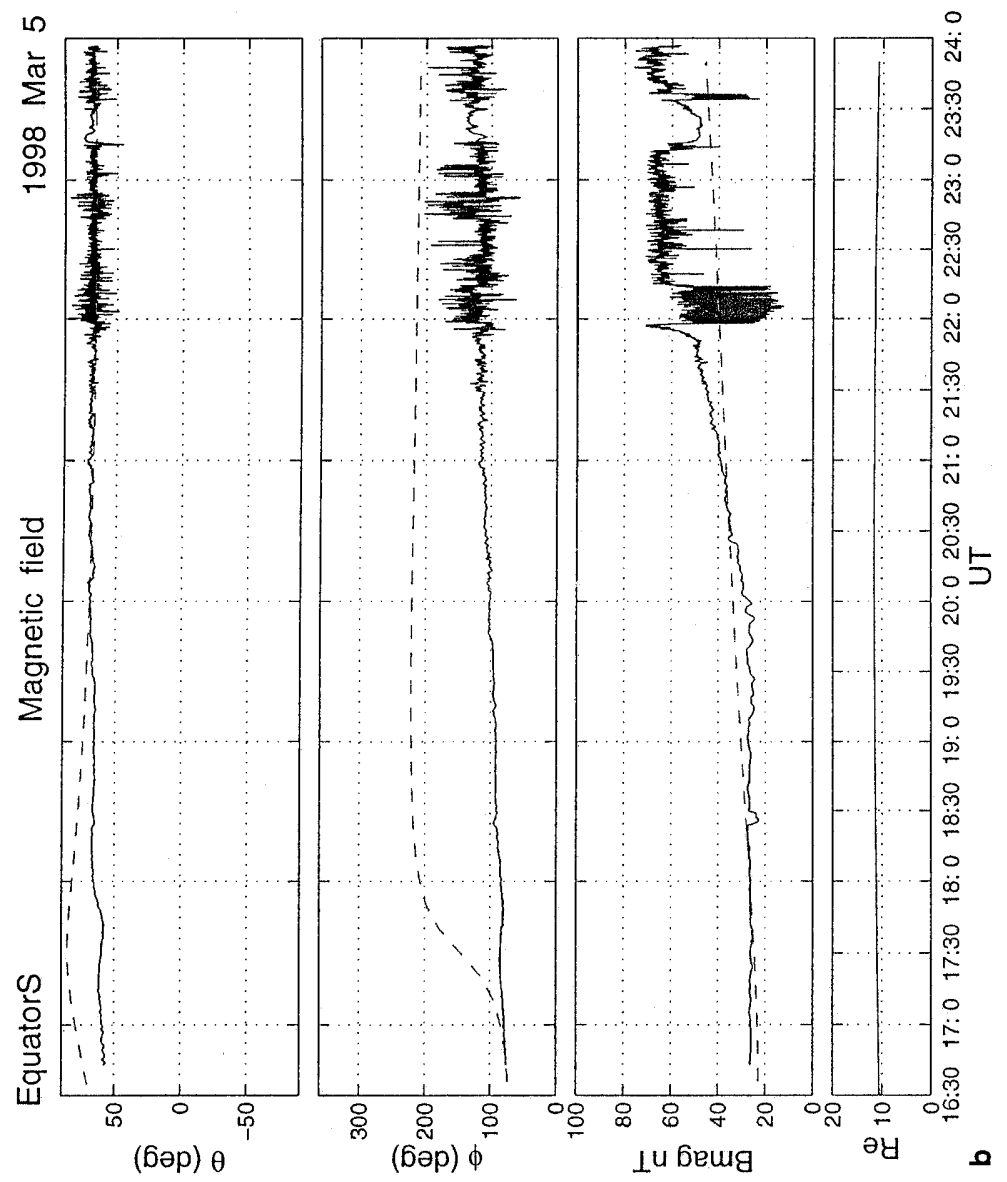

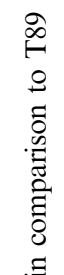

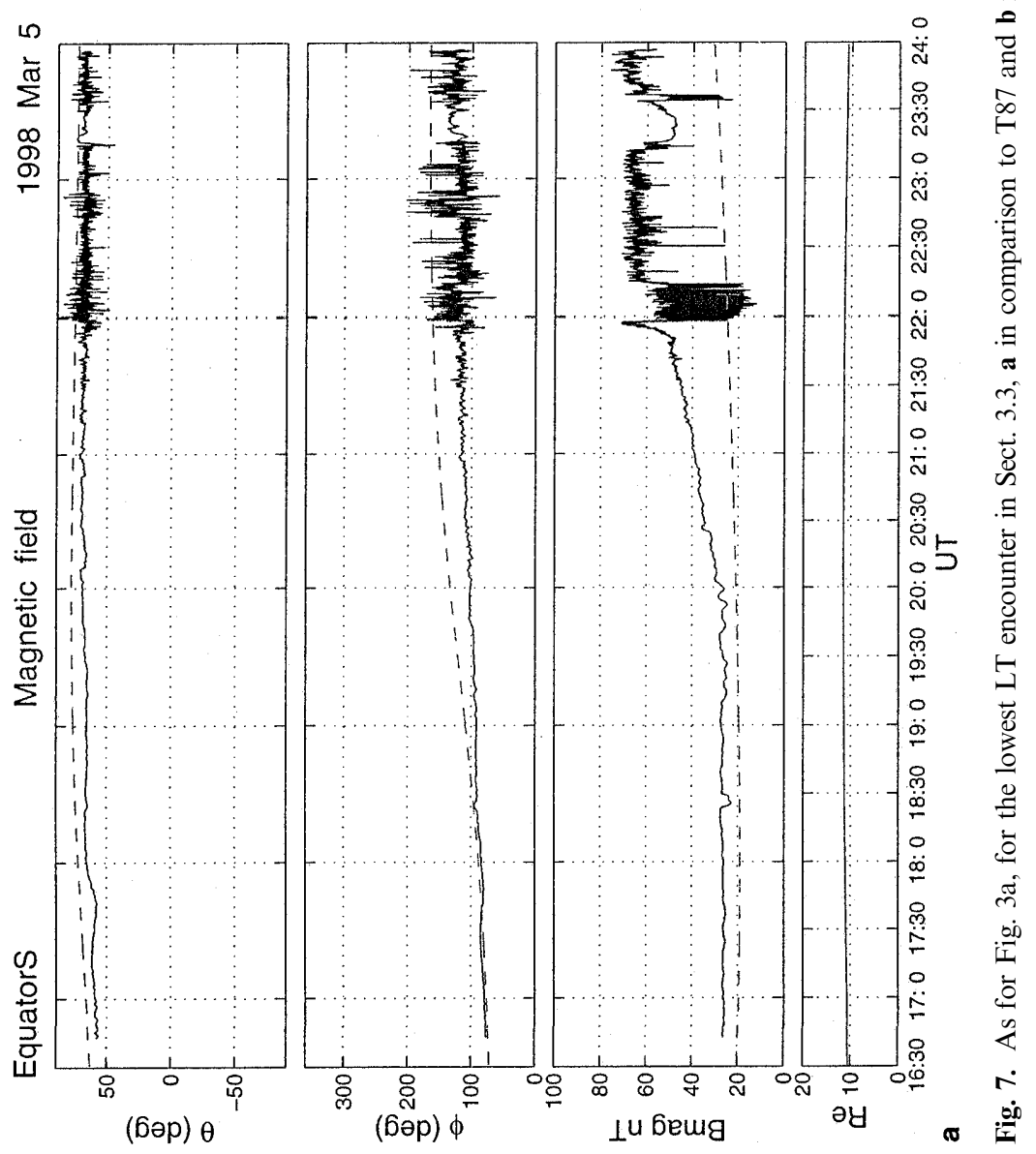


netic compression are high as well. Comparison with the data, however, shows similar agreement to the field orientation for both the T87 and T89 models in the case of 8 January, although the measured field shows a few degrees tilt closer to $Z_{\mathrm{GSE}}$, as might be expected for high ram pressure (Fig. 6a shows the T87 model). The 5 March data, however, does not agree closely with either field model, but is closer to the T87 model. This is discussed further in Sect. 4.1.

The lower panel of Fig. $6 \mathrm{~b}$ shows that the magnetosheath field is very closely aligned to the magnetopause orientation implied by the second encounter for the first event. Fluctuations of the field away from the magnetopause plane remain less than $\sim \pm 20^{\circ}$ and there is no evidence of a drift in the underlying magnetosheath field direction. It appears that large amplitude 'mirror' signatures, clearly occurring very close to the boundary in this case, do not affect any ordering with respect to the magnetopause boundary. This result is consistent with the other events containing strong mirror signatures. In fact, the two mean MVA normals at each encounter, given in Table 2, differ only by a few degrees, implying the boundary orientation is very stable.

Inspection of the model normals in Table 2 reveals that it is the change in the model boundary orientation between the two locations which results in the normal for the later encounter aligning better to the model boundary. All individual crossings behave as strong TDs and show similar scatter plots to that shown in Fig. 6b, with matched MVA and planar order. Individual crossings at the second encounter (10:50-11:15 UT) show evidence of small tilting, again predominantly in $(Z, Y)_{\mathrm{GSE}}$. Although these are $\sim 5^{\circ}$, the effect is apparent between magnetospheric field directions in the lower panel of Fig. 6b. It seems, therefore, that event is not controlled by the IMF direction, but predominantly by the high solar wind pressure, as is indicated by the orientation of the local magnetosheath field. The ram pressure increase is primarily a result of a rapid increase in solar wind density (for both events) and for 8 January, at least, this is consistent with both the common boundary orientation at the two encounters and a pressure sensitive crossing location.

The traces in Fig. 7, however, show that there is no field rotation across the magnetopause for the event of 5 March. The boundary analysis also shows good alignment of the magnetosheath field to the magnetopause orientation, with fluctuations which remain below $\sim \pm 10^{\circ}$. Although identification of other crossings is clearly difficult, there appears to be a re-entry into the magnetosphere between $\sim 23: 15$ and 23:35 UT. Furthermore, there is a sharp change in the magnetic signature at $\sim 22: 15$ UT, where the large amplitude compressional signatures abruptly cease.

\subsection{Long events with GTL conjunctions: January 6 and February 11}

The data intervals shown in Fig. 8 and 9 are chosen for illustration since they represent the longest events sampling the magnetosheath and contain magnetopause encounters separated by several hours. They contain high numbers of individual crossings and correspond to two events for which there was a close conjunction with the Geotail spacecraft. The encounters do not occur at extremes of the local time coverage, but they are separated by about one and a half hours of local time and cover a range of intermediate locations (from 07:0309:43 LT). The two events show very different characteristics, however: the 6 January event (Fig. 8) contains strong, well-ordered compressional fluctuations, whereas that for 11 February (Fig. 9) shows a mixed signature of multiple crossings. It is therefore of interest to compare the events in the context of the boundary analysis, but our primary aim is to combine the analysis at each spacecraft for the individual crossings, so as to compare the local behaviour of the boundary.

Data coverage for 6 January begins in the magnetosheath and the early encounters appear to result from a shallow re-entry into the magnetosphere, at only intermediate solar wind ram pressure which is consistent with the crossing locations. The later encounter (at 10:24 UT) represents a final re-entry into the magnetosphere and corresponds to a slight reduction of solar wind pressure. There is no clear IMF direction for the whole event, although the local magnetosheath field is consistent with an initially southward field, at an azimuthal orientation, perpendicular to the Parker spiral (see Table 2). The whole interval covers the $8 \mathrm{~h}$ period around apogee, which occurs just after 7:00 UT. The discontinuity at 14:15 UT corresponds to the arrival of an interplanetary shock and occurs on the inbound leg of the orbit. Solar wind conditions upstream of the interplanetary shock, measured by WIND, correspond to a ram pressure which would move the magnetopause inwards to nearly the Equator-S position at the time the discontinuity is seen.

The magnetopause encounter on 11 February provided a wide variety of crossings over several hours, also centred on apogee. Equator-S first exits the magnetosphere at $\sim$ 13:09 UT through a very complex boundary layer, following a highly unstable region near the boundary. The IMF is strongly southward at this time although the position of the crossing closely matches the ambient solar wind pressure (see Table 1). Both the ram pressure and mean magnetic compression at the boundary increase from intermediate values through the event. A series of distinct crossings follow until a slow re-entry into the magnetosphere at $\sim 17: 01$ UT. Each key crossing position is well matched to the corresponding solar wind pressure values. The earlier boundary layer crossing is the subject of another detailed study (this issue), so only the later crossings are discussed here.

Although the events occur during similar, high levels of magnetospheric activity, during the first (6 January) the level is decreasing and during the second it is increasing. Nevertheless, the 6 January event shows a very close match between the model field and the data, through the whole event, for both T87 (plotted in Fig. 8a) and T89; reminiscent of 8 January, 

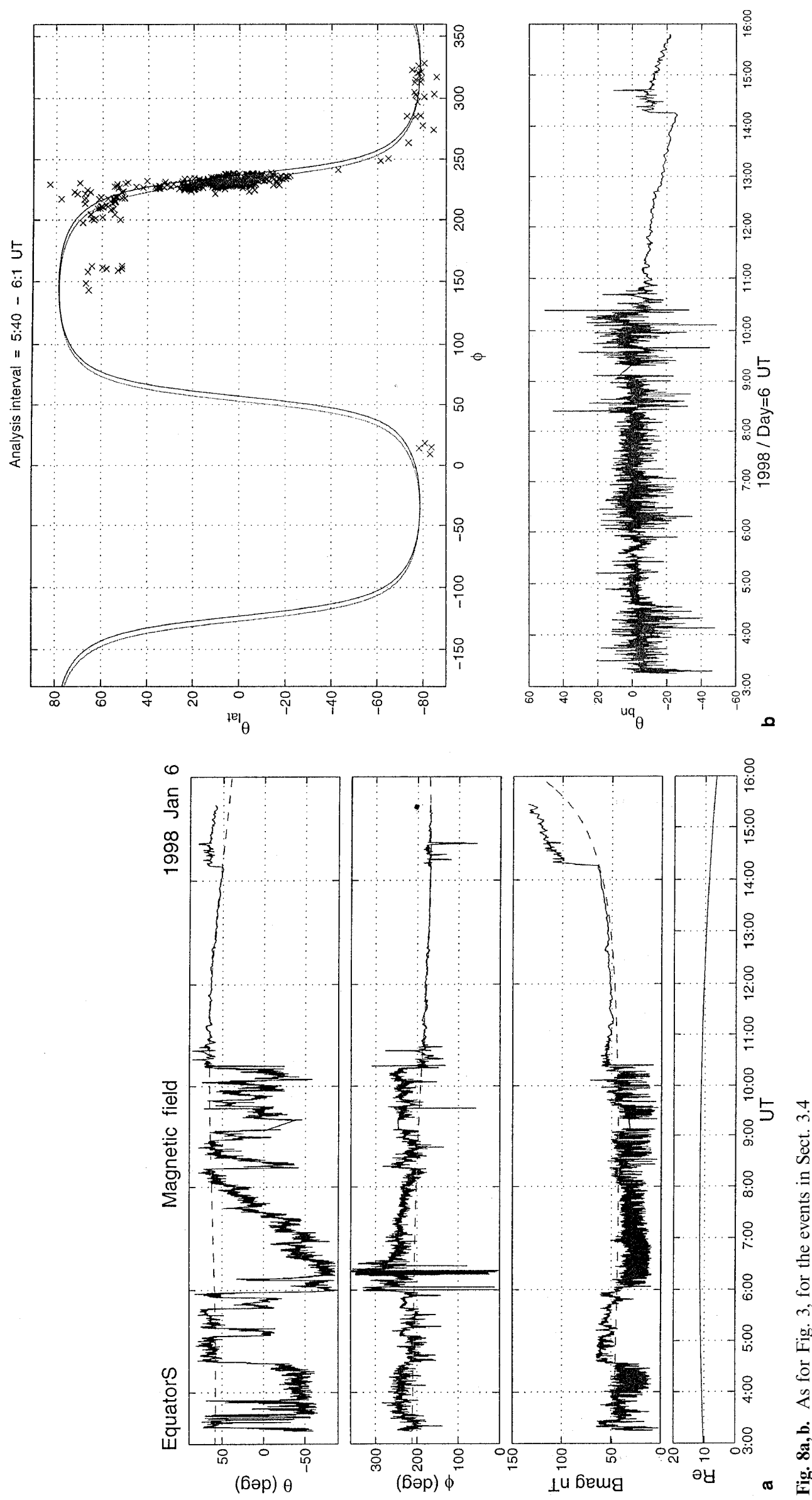

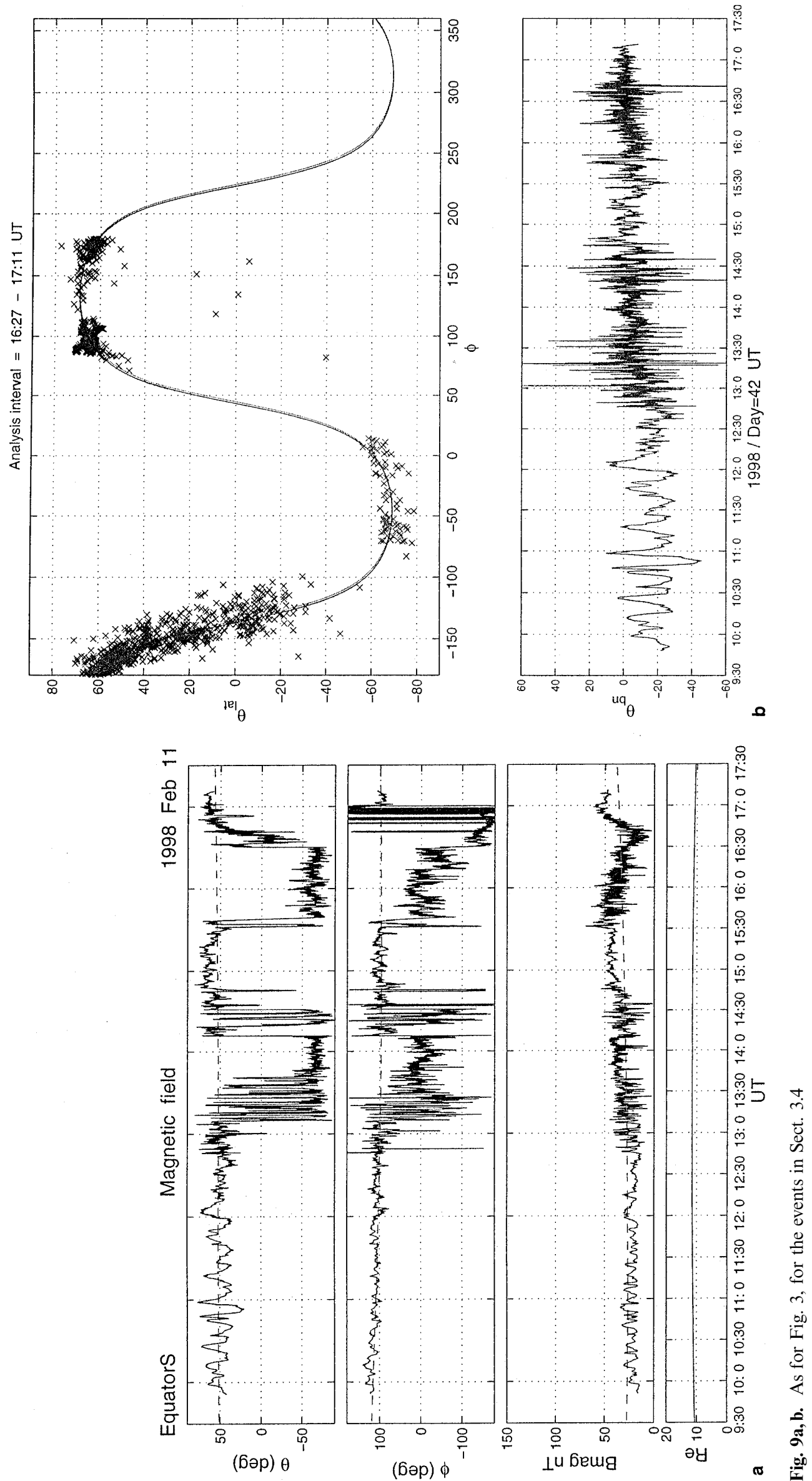
although then at high pressure. Note that the relevant local time is for the later encounter, at 10:24 UT, where the spacecraft finally re-enters the magnetosphere (i.e. $\sim 09: 43$ LT). For 11 February, the model field directions do differ, although the magnetopause field is too variable to clearly distinguish the effect with respect to the data.

The middle encounters during 6 January produce strong stable normals which show very little tilting with respect to one another. The scatter plots support the presence of an underlying planar field rotation (as evident in Fig. 8b, for the crossing at 05:52 UT) at all crossings, while suggesting the presence of additional structure at the later boundary. This later encounter $(\sim 10: 24$ UT) results in only a weak minimum variance direction, but its direction does correspond to the planar orientation, again suggestive of tangential ordering across the boundary. The implied MVA normal, however, is tilted somewhat Sunward $\left(\sim 5-10^{\circ}\right)$ of the normals for the other encounters, resulting in a slightly different magnetospheric orientation after the 10:24 UT crossing. As the lower panel of Fig. $8 \mathrm{~b}$ shows, this is again similar to the case of 8 January, in the sense that the magnetosheath field is well ordered by the earlier crossings between $4: 30$ and 6:00 UT, despite the long time scale (but distinct from the boundary orientation at the later crossing). The central crossings are where the
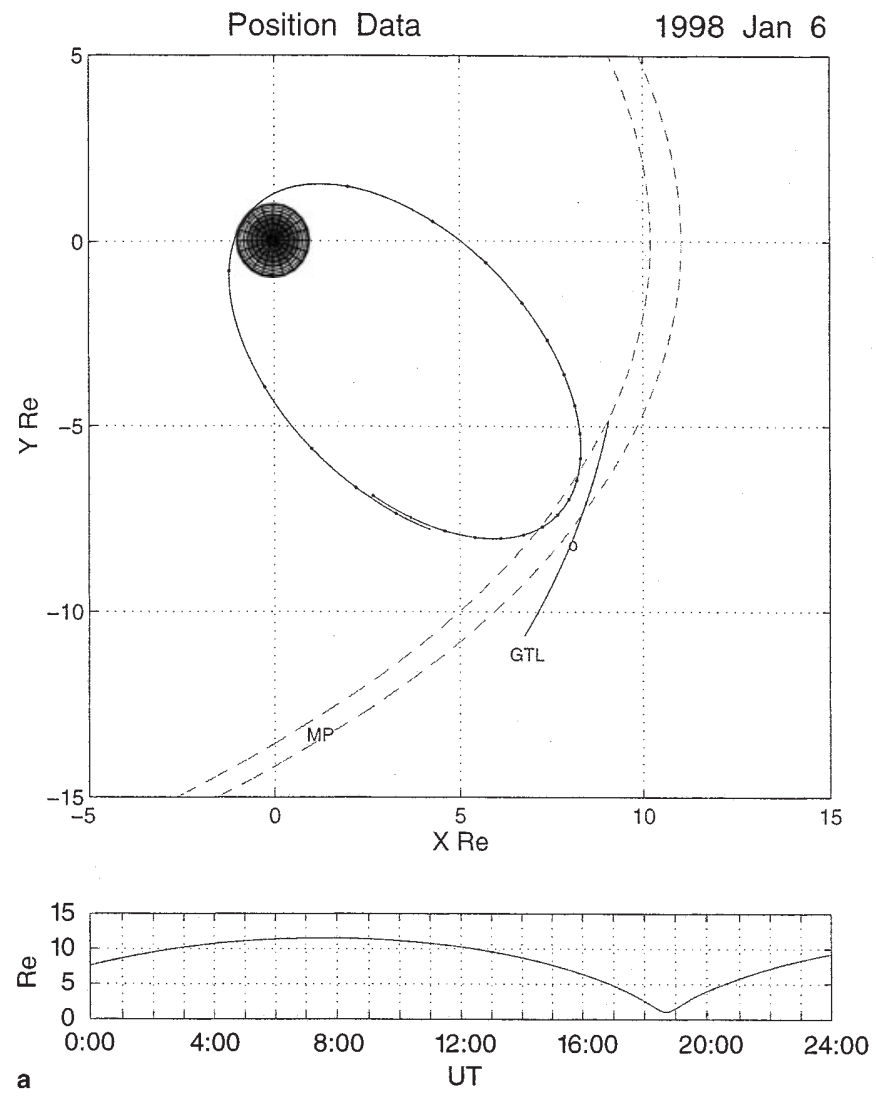

Fig. 10a, b. Geotail orbit segments and Equator-S for the time of the two conjunctions described in Sect. 3.4. The cuts through the relevant model magnetopause surfaces at the Equator-S crossings and in the $X, Y$ plane are shown, together with the Geotail position at the time of large amplitude compressional variations occur adjacent to the magnetopause.

Figure 9 gives the corresponding plots for the 11 February event. Despite the local magnetosheath field remaining strongly southward, the character of the crossings changes from earlier to later times. The second interval of crossings (between 14:10-14:50 UT) shows similar character to the boundary layer crossing, producing a non-planar distribution in the scatter plot of field angles, and different planar and MVA curves for the individual crossings. The two later crossing intervals (15:30-15:40 UT and the slow encounter between 16:3017:10 UT), on the other hand, show strongly tangential order through the magnetopause (with matched MVA and planar curves). The slow crossing is illustrated in Fig. 9b, revealing that the magnetosheath is only well ordered around the time of the later crossings. The plot of the field orientation in the lower panel highlights the more Sunward orientation of the initial magnetopause encounter (at 13:09 UT) and is clearly consistent with magnetic erosion, producing a blunter magnetopause. Apart from the change in normal direction between the early and later magnetopause encounters, the individual crossings do not show significant relative tilts.

Figure 10 shows the orbit conjunctions with the Geotail spacecraft for both events, projected into the $X, Y_{\mathrm{GSE}}$ plane, where radial distance is also given only
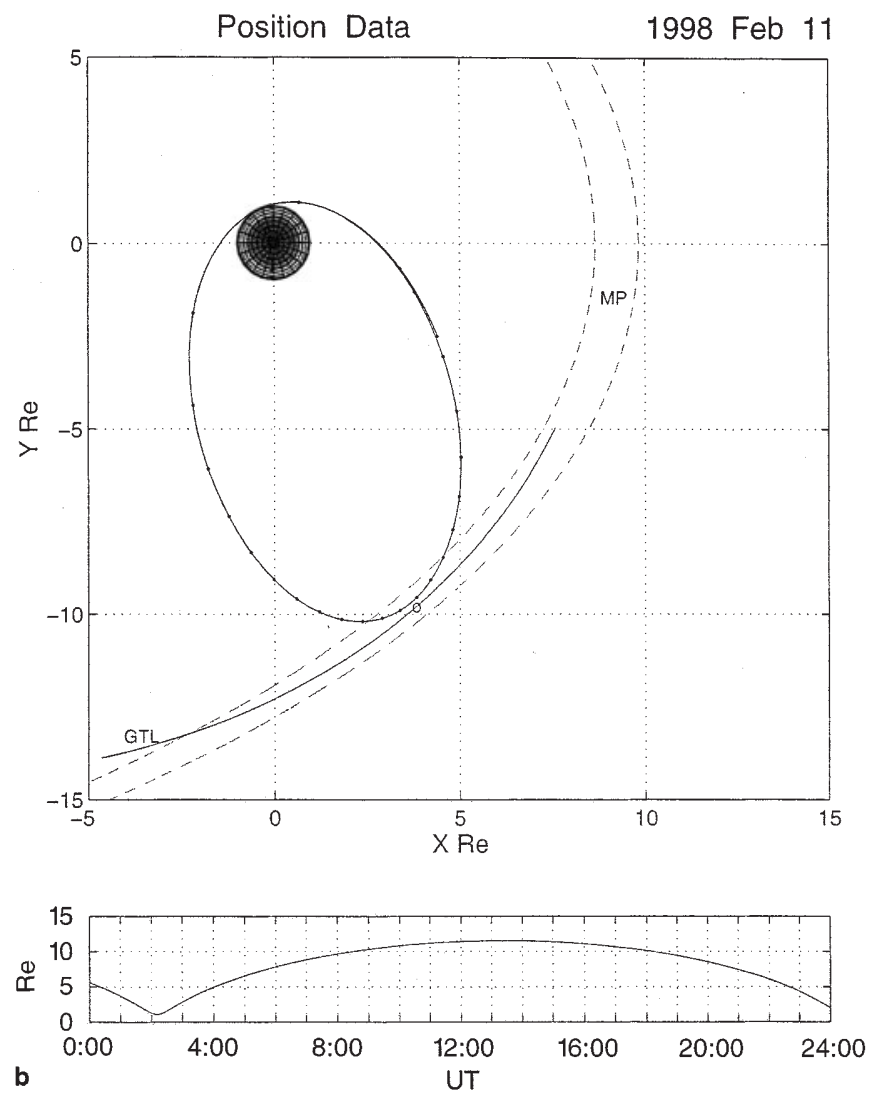

closest approach (circle). Note that Equator-S moves anticlockwise around the orbit and the lower panel gives radial distance from the Earth over the day 
for Equator-S. The small, filled circles on the orbit tracks of Equator-S correspond to hour intervals, starting with 00:00 UT on the day. For 6 January (Fig. 10a), closest approach occurred at $\sim 06: 00$ UT. Note that the Geotail orbit (labelled GTL on the figure) lies close to the ecliptic plane $\left(X, Y_{\mathrm{GSE}}\right)$ and is plotted for the time segment from 04:00-08:00 UT. Also plotted are cuts through the model magnetopause surfaces for the fits given in Table 1, at both the $Z_{\mathrm{GSE}}$ heights of Equator-S and Geotail (outer curve). These therefore represent the position of the magnetopause at the time of the Equator-S crossing at 05:52 UT. For 6 January, the magnetic field data for Geotail indicates the spacecraft may briefly enter the magnetosphere (although remaining only in the boundary region), and then finally exits into the magnetosheath at $\sim 05: 40$ UT. This crossing time, however, is $\sim 15 \mathrm{~min}$ before Equator-S and is indicated in Fig. 10a as a circle on the trajectory. The cuts in Fig. 10a show that the model boundary would clearly cross Geotail before Equator-S. Thus, an earlier crossing time at Geotail is consistent, since the boundary normals found for both spacecraft closely align with the model boundary orientation. The timing of the crossings put an upper limit on the magnetopause speed at about $10 \mathrm{~km} / \mathrm{s}$.

For 11 February, the Geotail trajectory is drawn for the time segment from 09:00-19:00 UT in Fig. 10b, where closest approach occurred at $\sim 15: 30$ UT. The magnetic field data for Geotail is plotted (grey trace) together with Equator-S data in Fig. 11 and shows an exit into the magnetosheath after Equator-S, at 15:50 UT, and a re-entry into the magnetosphere just before
Equator-S, at $\sim 17: 01 \mathrm{UT}$. There is a coincident partial crossing at 15:33 UT, the approximate time of closest approach, and this is indicated by the circle on the Geotail trajectory. The model magnetopause cuts are shown for the fit corresponding to the Equator-S crossing at 17:01 UT (note that this is the later encounter with respect to the time of the conjunction at $\sim 15: 30$ UT) since it is clearer to choose only one of the encounters seen at the spacecraft. Despite Geotail remaining outside Equator-S, as before, this time the model remains outside the orbit at Geotail, so that an inward moving magnetopause will cross Geotail later than Equator-S and vice versa. Moreover, the orientation of the Geotail orbit remains nearly parallel to the magnetopause, so that similar boundary crossings are observed, as is clear from the superposed data plot (Fig. 11).

It is also clear that the relative crossing times are again consistent, since the MVA normals again approximately align with the model boundary (at least at the slow encounter at 17:01 UT). The partial crossing at 15:33 UT, however, shows a significant tilt, sunwards, away from the model orientation at both spacecraft and this can account for the nearly coincident crossing at this time. In fact, the MVA normal at Geotail is oriented $\sim 25^{\circ}$ sunward of the model normal for the crossing at 15:50 UT and this has the effect of increasing the interspacecraft time difference at the 15:50 UT Geotail crossing, where Geotail lags Equator-S. It is possible the magnetopause is rippled (by a surface wave) during this crossing. Since the Equator-S normals do not change as much as the Geotail boundary normals, the amplitude of any ripple is much smaller at Equator-S. It is
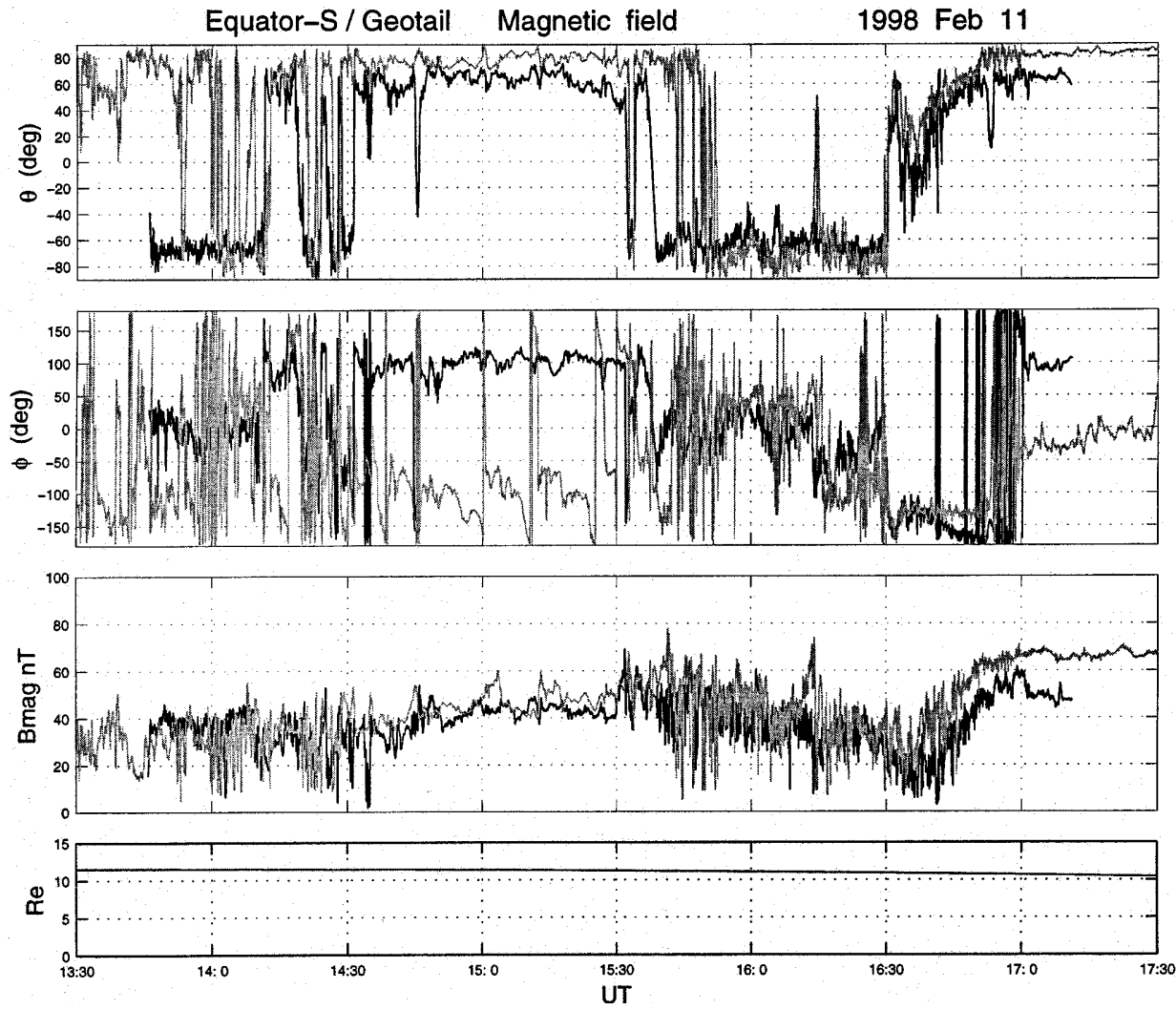

Fig. 11. Comparison of Geotail (light trace) and Equator-S magnetic field data, for the event in Fig. 9 
therefore not possible to make reliable estimates of motion along the magnetopause. Nevertheless, Sunward turning of the normals at the earlier crossing is consistent with an anti-Sunward moving depression. The inward speed of the magnetopause at the 17:01 UT crossing is harder to estimate from the timing, since the spacecraft are not radially separated, but appears to be a little faster $(\sim 10-20 \mathrm{~km} / \mathrm{s})$ than that of 6 January.

\section{Categorisation of the crossing set}

It was argued in Dunlop et al. (submitted to JGR) (and partly in Sect. 2) that the majority of the observed magnetopause traversals, together with their magnetosheath data, showed a number of features which were consistent with the spacecraft remaining near to the magnetopause during its exit into the magnetosheath. Section 2, for instance, found that most crossing positions approximately scaled with ram pressure and that the magnetospheric field often reflected a corresponding level of local magnetic compression. Furthermore, clear control of crossing position by southward IMF (during low-pressure conditions) could be established. Both led to the conclusion that nearly all magnetopause passes were associated with encounters at or near stable locations of the magnetopause, implying no large-scale motion of the boundary occurred away from the spacecraft locations. This view is further supported by the result that for most magnetosheath intervals the background field direction remains well aligned (less than $\sim 10^{\circ}$ ) to the mean boundary orientation inferred by the calculated normals. This alignment is indicated by ' $\mathrm{Y}$ ' in the first of the columns headed 'MSH order' in Table 2 when it extends over the whole of the measured interval. The magnetosheath field is therefore closely draped, tangentially to the magnetopause boundary and the implication is that most magnetosheath excursions are shallow; consistent with the expectation that the underlying magnetosheath field orientation will deviate from that at the boundary (Southwood and Kivelson, 1995).

This implication is reinforced by the fact that few passes do show evidence of deeper excursion into the magnetosheath, as discussed for the events earlier. It is clear from Fig. 1, however, that the events occurring at early local times $(<09: 00$ LT) have apogees located within $\sim 1 R_{E}$ of the crossing locations. For the other events, the spacecraft has to sample the magnetosheath near apogee while under solar wind conditions of either high ram pressure or strongly southward IMF, in order that the magnetopause occurs a few $R_{E}$ distant. In fact, very few passes satisfy these criteria, even marginally and there is no guarantee that the magnetopause will remain deflated, following a close crossing, outbound, or prior to a close crossing, inbound. Of the inner crossings shown by the plot in Fig. 1, two days stand out (7 January and 8 January). The 8 January event has been discussed and the 7 January, inbound, does indeed show a small drift of the magnetosheath orientation, relative to the magnetopause boundary.
The second column under 'MSH order' in Table 2 indicates (by ' $\mathrm{m}$ ') when the magnetosheath field clearly developed strong, compressional signatures, over all or part of the interval, having magnetic characteristics which are consistent with mirror modes. The indication ' $\mathrm{m}$ *' for 16 December event and others is given since the signature did not lie close to the magnetopause (as shown in Fig. 3). These occurred for $30 \%$ of the passes and often were of extended duration (hours). All magnetosheath excursions but one correspond to the well ordered (draped) magnetosheath intervals and most begin immediately adjacent to the magnetopause crossing. In fact, it is clear that all the intervals identified as reflecting the presence of possible mirror mode structures follow the magnetopause very closely, despite the presence of intervals containing other magnetosheath signatures, a few of which do not. Nevertheless, a number of additional passes are also listed which show close alignment to the magnetopause boundary orientation. In all the examples discussed, the field remains well within $10^{\circ}$ of $\theta_{B n}$, with fluctuations only rarely exceeding $20^{\circ}$, and this is typical of the data set.

Apart from its tangential alignment, the magnetosheath field orientation resulted in large magnetic shear relative to the magnetospheric field at the magnetopause, although a range of orientations occurred. To some extent magnetic shear controls the character of the magnetopause, amongst other factors. Nevertheless, there is also likely to be a local time dependence. Near the magnetopause, moreover, the magnetospheric field may deviate from its nominal direction as well as exhibiting enhanced magnetic compression under certain conditions. In this respect, comparison to the model field is of interest. Both the nature of the magnetopause crossings themselves and the implied configuration of the magnetospheric boundary field with respect to the Tsyganenko field models are therefore discussed in the sections next.

\subsection{Model comparisons: global field and magnetopause boundary}

The comparison of the magnetic field orientation to the model field has been partly illustrated by the selected events described and Figs. 12 and 13 provide further illustration. In all cases, the model magnetic field is computed from the combined contribution from both the external current systems defined in the Tsyganenko models (Tsyganenko and Usmanov, 1987; Tsyganenko, $1989)$ and the IGRF magnetic field. Where appropriate, any effect of the model dependence on $K_{P}$ has been evaluated. Although there is clearly a wide range of solar wind pressure and IMF conditions pertaining during the set of crossings, only a few events show high $K_{P}$ values and do not appear to account for the trends observed. Since the purpose here is not to quantify actual deviations of the models for given $K_{P}$ states, we have intentionally compared the earlier field models, which are relatively insensitive to external parameters, usually for average activity levels, rather than the more recent field model. 

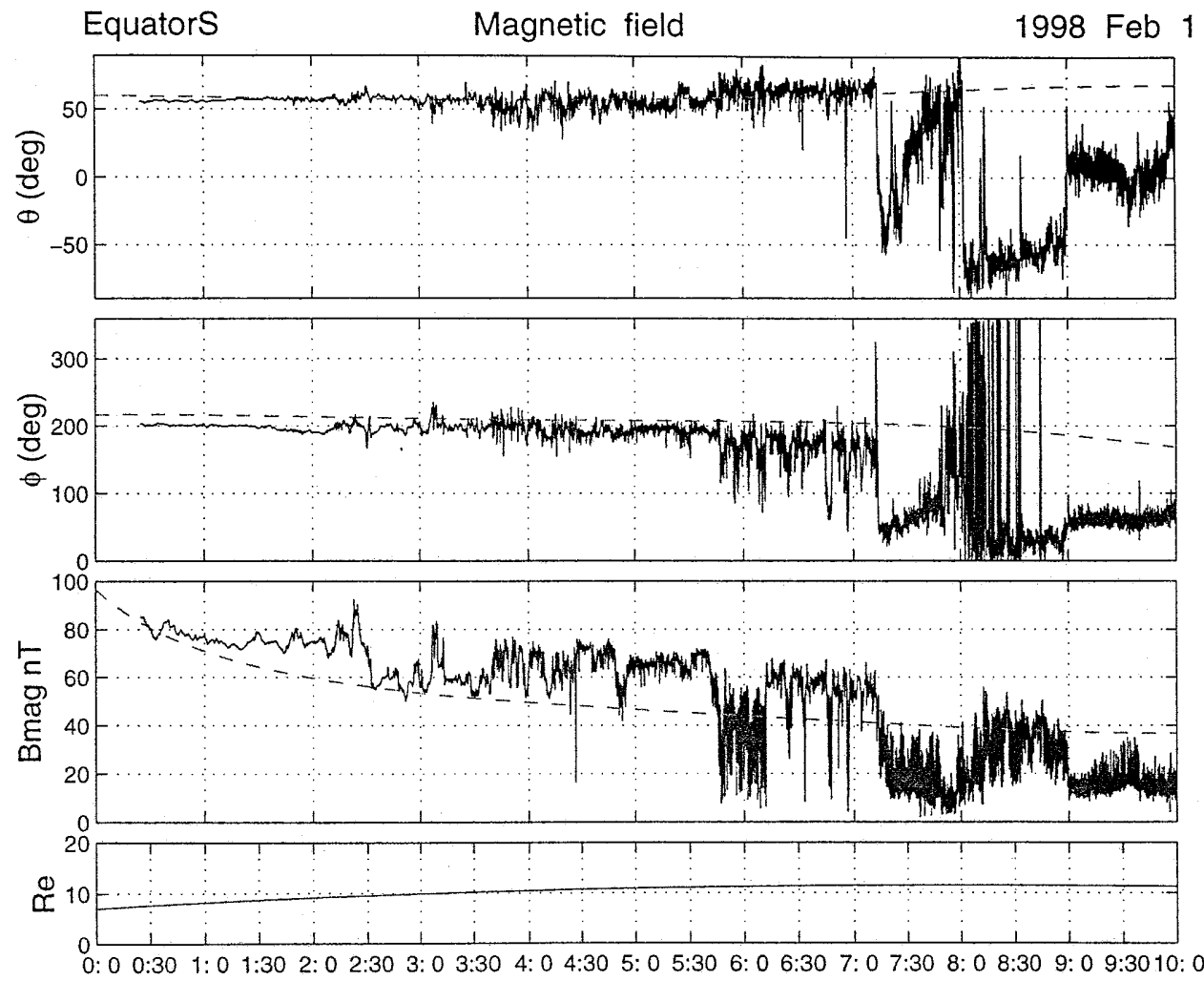

UT
Fig. 12. As for Fig. 5a, for the T89 model
The magnetic field is not expected to exactly correspond to the model field, of course, particularly near the magnetopause, where magnetospheric state is most sensitive to the response to external conditions. The interest here is therefore rather in the systematic differences between the two basic field models of Tsyganenko (T87 and T89) in relation to the measured field, in order to highlight the local field configuration observed in the equatorial dawn-side region. Thus, although, to some extent, the configuration of the magnetospheric field will depend upon the local conditions, it nevertheless appears that there is a systematic trend in the deviation between the direction of the magnetic field for each model and the data (near the magnetopause) which worsens with decreasing local time. This angular difference is of order $10^{\circ}$, typically, but can be greater. Such deviations are not so remarkable, but the fact that they are systematic is significant. It is also important to note here that although the measured field exhibits magnetopause crossings in these events, the implied magnetopause in the model lies outside the spacecraft positions in the majority of cases, and always does for the examples quoted.

For the passes corresponding to high local times (greater than about 09:00 UT), there is typically very good agreement in the field orientation (typically $\sim 5^{\circ}$, or less) between the models and the data, despite an enhanced, local magnetic compression often being present. The field models are usually indistinguishable in their orientation, although as would be expected they do show a sensitivity in the field magnitude to $K_{P}$. The selected events in Sect. 3 already illustrate this, with only the events corresponding to lower LT showing significant angular deviations. The best confirmation of the agreement at high local time is via the events of 16 December and 8 January. The magnetopause encounter on 16 December shows the effects of southward IMF, whereas the event on 8 January shows very high magnetic compression as a result of extreme solar wind ram pressure. Nevertheless, for both there is little deviation between the calculated model fields for each model, as described in Sect. 3. The small deviation shown for 8 January is consistent with the effect of a highly deflated magnetosphere, but is similar for the two models.

By contrast, Figs. 5a and 12, Figs. 7 and 13 show the comparison between the T87 and T89 models for the three passes on 1 February, 5 February and 5 March, corresponding to decreasing local times ranging from about 08:00-06:00 LT. Figures 5a and 12 show the comparison between the two models for the event on 1 February which is typical of passes locate around this local time (see Table 1). The T89 model field remains at a greater azimuth angle than the measured field although the T87 shows good agreement. For other events, the T89 field can also show a greater latitude angle than the T87 and the data. Note that the azimuthal field direction suggests that the spacecraft lies above the magnetic equator for this event and this is supported by the equivalent plots in GSM co-ordinates. In fact the sense of the deviation changes depending upon whether the spacecraft lies above or below the magnetic equator for other events and suggests that the effect is related to the form of the equatorial current 

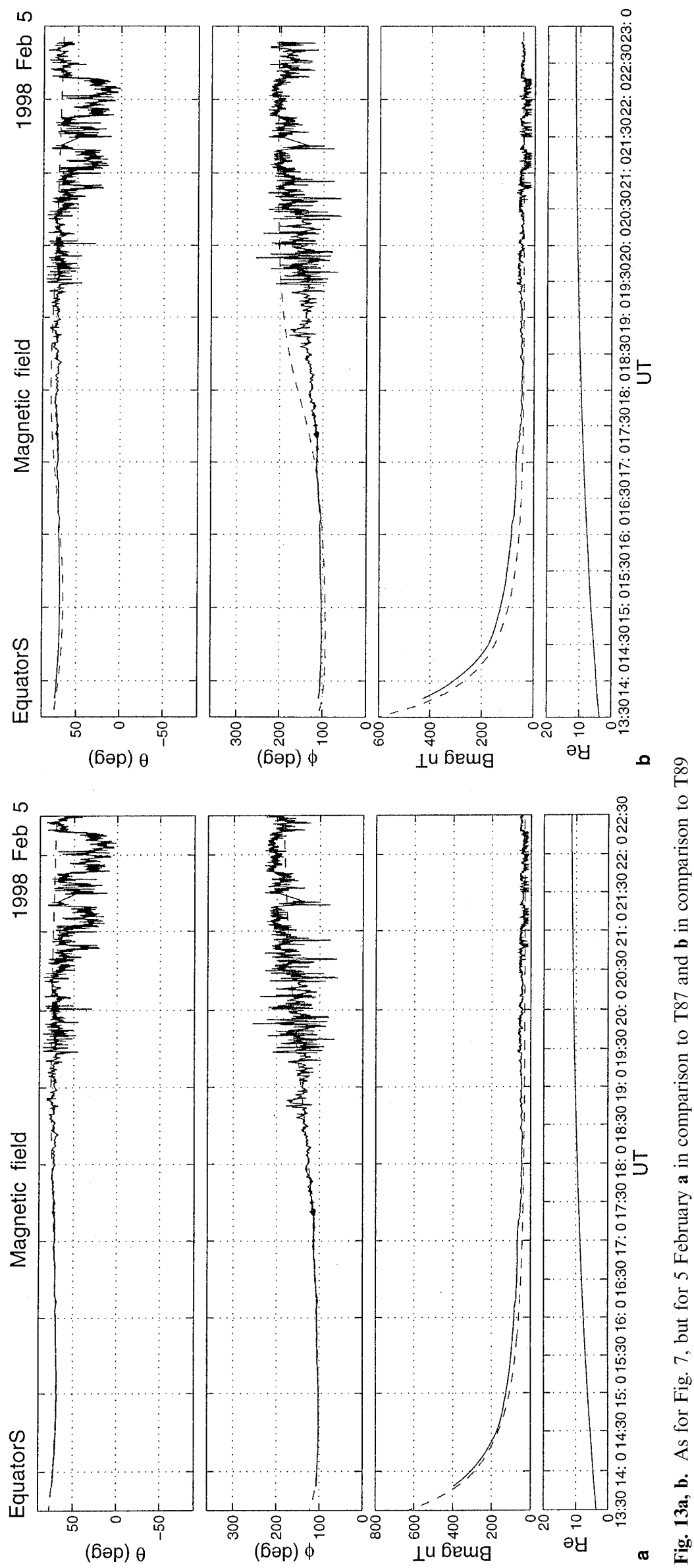
systems in the two models. With respect to the data, the global implication is that the magnetopause is less flared than suggested by the T89 model, but this is not consistent generally with the observed magnetopause orientation. It would seem, therefore, that the effect is more local to the dawn-side equatorial region.

The two other events, 5 February and 5 March, which are separated by nearly one hour of local time, clearly reflect the effect of the spacecraft passing from above to below the magnetic equator. For 5 February, at 07:10 LT and simply controlled by solar wind pressure, conditions are quiet, and there is little local magnetic compression at the magnetopause. The T87 model follows the data extremely well, whereas the T89 predicts an early traversal of the current sheet, prominent as an azimuthal turning in the field. Such an effect in the T89 model would be consistent with some warping of any equatorial current extending into this region out of the plane of the magnetic equator and this could arise from the influence of the model tail current sheet. This is a key difference in the T89 model over the T87 model and although designed to better account for the form of the magnetotail field, it remains warped on the flanks at positions near the dawn/dusk terminator. It is possible, therefore, this arises from an effect of the different form of the tail current sheet in the T89 model which does not seem to be reflected in the data.

In fact, the large discrepancy between the models in the region covered by 5 March again results from a different field configuration across the magnetic equator (not reflected in the data), just before the magnetopause crossing. The data suggests that the spacecraft remains above any azimuthal (ring) current, while both models imply it should traverse any ring current. Although both models deviate somewhat from the data, it is more prominent, and occurs earlier, for the T89 model. Although this pass corresponds to higher activity levels $\left(K_{P}=4-\right)$, the enhanced effect is a result of the lower local time (06:07 LT) and supports the possibility that the flank region is not well modelled by the form of the T89 warped tail current sheet. Apart from this effect in the local dawn-side region, it is remarkable how closely the data generally follows the model field orientation over a wide range of local and solar wind conditions.

\subsection{Boundary properties}

The orientation of the boundary has been estimated here primarily using minimum variance analysis (Sonnerup and Cahill, 1967), in combination with an investigation of the planar order of the magnetic field through the boundary, in an attempt to identify the character of the boundary crossings and any fluctuations in boundary orientation at the individual crossings, as described in Sect. 3. The discussion in Sect. 3 included an account of the boundary analysis performed for each event, selected to illustrate the varying character over the crossing set. The analysis for all the events, identified by the key crossings as in Table 1, is summarised in Table 2 which shows the mean, or key, MVA normals, calculated over the associated crossing intervals. These have been compared to the model boundary normals, calculated from the fitted model surfaces for each crossing. In addition, the relative orientation of the individual boundary crossings, associated with each listed encounter, is indicated. We also descriptively characterise each event by noting: the nature of the boundary at each key crossing, the orientation of the individual crossings where multiple crossing encounters occur, and some details on the magnetosheath features and order with respect to the boundary.

The MVA results are summarised following the column headed 'MSH order', which briefly indicates the magnetosheath configuration as discussed already. The minimum variance vector, expressed as components in GSE co-ordinates, and the ratio of minimum to intermediate eigenvalues is given. The following column shows the model boundary normal, at each crossing position (listed under $\mathrm{x}, \mathrm{y}, \mathrm{z}$ ), to the fitted magnetopause surface (taking into account either IMF or ram pressure dependence, as discussed in section 2). Most of the calculated boundary normals are well established by eigenvalue ratio and deviate somewhat from the model normals: lying Sunward of the model orientation in nearly all cases. The two columns under 'Orientation' give the angle between the model and MVA boundary normals and indicate when the calculated normals differ by a little more than $20^{\circ}$ in orientation. This result has not been confirmed for the couple of cases where MVA did not give stable normals. Also, both the presence of extreme pressure conditions (v. high $P$ in the table) or relative tilting of the normals in $Y, Z$ ( $z$-tilt) have been indicated. Most normals, however, have a $Z$-component which is consistent with the model boundary, but deviate in $X, Y$. Thus, although the variation in both model and calculated normal directions over the whole set of crossings is as expected, approximately following the model boundary surface orientation which changes over the range of crossings by more than $60^{\circ}$, a Sunward orientation occurs for the large majority of the passes.

This result is in contrast to the close agreement found between $P_{\mathrm{MP}}$ and $P_{\mathrm{ram}}$ for the crossing locations (as well as the agreement to observed $B_{Z}^{\mathrm{IMF}}$ ) and supports the conclusion that the measured normals consistently suggest a more flared, or blunter, shape to the magnetopause than indicated by the Sibeck et al. (1991) model, rather than arising from poor knowledge of magnetospheric state, poor identification, or other errors. In particular, the effect of IMF direction on the model shape has already been taken into account in the fits to location and in any case accounts for only a few degrees of deviation in normal direction at these dawn-side locations. This suggests the effect of magnetic erosion on magnetopause shape is insufficient to explain the orientations found and in fact the local magnetic shear (see Table 1) shows a range of values which are not well correlated with the deviations between the model and MVA normals.

Although detailed analysis has not been completed except for the events in Sect. 3, most boundary normals within a pass and for each key encounter show very little 
variation from each other and depend predominantly only on location (LT). Furthermore, where multiple crossings are observed (particularly for the long events) there is, rather unexpectedly, little evidence of large local changes in boundary orientation, or any which would be consistent with boundary waves or pressure ripples. This latter result is consistent with the view that the crossings arise predominantly from small fluctuations of the magnetopause close to the spacecraft, rather than large amplitude changes from far away. It may also be a consequence, however, of the presence of the ordered, compressional signatures ('m' under 'MSH order') in most long intervals of magnetosheath data and further work is needed to clarify this. Only the special case of the Geotail events, as described in Sect. 3.4, suggests a wave like boundary ripple is present. The changes are still only marginal at Equator-S, however, but are reflected by larger amplitude tilts at Geotail, consistent with an anti-Sunward moving depression.

Where small changes in direction are found between the individual magnetopause crossings, these can be identified with the presence of a more complex boundary structure rather than with large amplitude fluctuations in boundary orientation. The columns headed ' $\Delta \mathrm{n}$ ' and 'MP' indicate the events where significant, small differences between the individual crossing normals were found and confirm whether the boundary exhibited tangential field rotations through the boundary (TD), respectively. A notable addition to the events in Sect. 3 is the pass on 7 January, listed in Tables 1 and 2, which provides strong MVA crossing normals, having $\sim 10^{\circ}$ relative orientations with respect to each other, but with a miss-aligned magnetospheric field (by $\sim 20-30^{\circ}$ ), just inside the magnetopause. Of the examples analysed, only the pass on 1 February contains significant relative tilting, while appearing to show strong tangential order. This is the pass which was identified as representing unstable conditions in Sect. 2, however, and it is likely that the magnetopause is unsteady at this time. The other unstable event, 4 January, also shows tangential order, although the magnetosheath field is southward.

In fact, for most of the early passes, the implied IMF direction (usually matching the local magnetosheath direction) corresponds well to the nature of the magnetopause boundary, so that strongly southward orientations (or reversals in the IMF direction) do not exhibit TD character. Although the pass on 9 January, at 10:05 UT, occurs during southward IMF, at the magnetopause its local magnetosheath field is not. For earlier local time $(<09: 00 \mathrm{LT})$, more of the crossings tend to have TD structure and do not correspond well to the local magnetic shear. It is possible that further into the flank region the effect of magnetic shear at the magnetopause is less than at local times close to the subsolar point. Typically, for the dawn-side events, a magnetosheath transition region is not apparent in the magnetic field signature, with immediate exit into often large amplitude magnetic compressions being a feature of the magnetosheath field. The magnetosheath signatures in this region are as likely to have been convected with the magnetosheath flow along the magnetopause, as to be a reflection of local magnetopause response and this may account for the lack of close correspondence between magnetopause character and the magnetosheath field. It should be remembered, however, that the events at lower local time tend to occur at higher ram pressure conditions.

\section{Conclusions}

We have performed a survey of all magnetopause encounters by the Equator-S spacecraft, using only the magnetic field data taken by the MAM experiment, in order to categorise magnetopause location and properties and the associated magnetosheath behaviour in the equatorial dawnside region. In all, 131 crossings on 31 orbits provide long duration coverage of the near magnetosheath, adjacent to the magnetopause, from 06:07 to 10:43 LT. Such extensive coverage in this region is uncommon. Selected encounters have been discussed in some detail, representing the extent of the local time coverage, and the boundary properties of the individual crossings have been summarised (in Tables 1 and 2), along with a catalogue of the key magnetosheath features. It is clear from a number of indicators discussed in the text, that the large majority of the encounters occurred during stable magnetopause locations, and that the spacecraft remained in the transition region and near magnetosheath. As a consequence, many encounters contained a high number of individual boundary crossings. The study is clearly limited by the lack of routine coverage by other experiment data and we have therefore enhanced the identification by routinely comparing the data to the T87 and T89 magnetic field models. Certain characteristics have been found, however, which we feel primarily result from behaviour in the dawnside region.

In Sect. 2, the occurrence and locations of the crossings were compared to solar wind pressure and IMF directions using a simple model of the magnetopause boundary. Where solar wind conditions (of $P_{\text {ram }}$ and $B_{Z}^{\mathrm{IMF}}$ ) are known, we find that the required solar wind parameter to best fit the key crossing positions generally uniquely matches observed conditions. The majority of crossings in fact scale with ram pressure. The effect of apogee distance as the orbit evolves further into the flank region, is therefore to increasingly bias crossing occurrence to conditions associated with intermediate, rather than low, solar wind dynamic pressure. Nearly all the crossings were thus found to correspond to either pressure balance response or the effects of strongly north/south IMF, as manifested in the magnetosheath; very few corresponding to a combination both effects. In the majority of cases, the local magnetic compression at the boundary was consistent with expectation from either of these controlling factors.

The boundary analysis for most encounters yielded stable normals, which rarely showed significant relative tilting between the individual crossings. Where tilting occurs, it can usually be attributed to more complex boundary structure usually under conditions of high 
magnetic shear at the magnetopause, in line with expectation (but see later for the crossings at low LT). The lack of large, local changes in boundary orientation suggests that the crossings do not result from large amplitude fluctuations in magnetopause position or from large amplitude pressure ripples on the boundary.

In contrast to the locations, however, the observed magnetopause orientation (determined by MVA), although broadly consistent with predicted boundary normals, typically lies Sunward of these. The variation in direction of the boundary normals over the set of crossing locations is large $\left(\sim 60^{\circ}\right)$, for instance, whereas the difference between the calculated and model normals remains systematically about $20^{\circ}$. Although there were a number of crossings corresponding to clearly observed, southward IMF, which were fitted to a blunter magnetopause, as described in section 2, these did not account for all the differences between MVA and model normal orientations. The remaining crossings, furthermore, did not show any strong biasing to high magnetic shear conditions. This implies that in this region the dayside magnetopause is blunter than that predicted by the Sibeck et al. (1991) model for the particular ambient conditions, even though crossing locations are well predicted. Indeed other effects, such as the strong draping of the magnetosheath field and regular presence of strong compressional signatures, may be responsible for the bias in magnetopause orientation.

Selected, individual passes were discussed in Sect. 3, including the detail of their boundary characteristics. These illustrate both the general properties seen in the data set, and the range of conditions encountered, in terms of: solar wind pressure, magnetospheric compression, magnetic shear at the magnetopause, and magnetosheath features. Some long intervals of a few to several hours, and containing a high number of repeated crossings, were observed, providing extended coverage of the near magnetosheath. A few encounters, exhibiting unusually high magnetic pressure states, were also found. Of particular note is the presence of large amplitude compressional signature, often adjacent to the magnetopause and without an apparent transition layer. Many of these show features corresponding to mirror-like signatures in the adjacent magnetosheath during a significant fraction $(\sim 30 \%)$ of the encounters. Overall, most encounters exhibited good tangential ordering of the background magnetosheath field to the magnetopause orientation throughout the intervals, consistent with strong draping and/or proximity to the magnetopause, with only a few exceptions. Although at the subsolar point the magnetopause and bow shock are approximately aligned, the boundaries are not near the flanks. More importantly, the background field direction generally need not change through the magnetosheath to be parallel to the magnetopause at the subsolar point, but typically does near the dawn-side flank in order to take the solar wind orientation.

The event of 16 December illustrates that a minority of passes, positioned at relatively high local time (09:0010:40 UT), show magnetopause encounters exhibiting properties which have been commonly observed before and which are clearly controlled by, or at least consistent with, their local magnetosheath conditions. The majority of passes, however, occurring further into the dawn-side region, tend to show more unusual characteristics. For example, where the magnetopause crossings behave as strong tangential discontinuities, typically there is a correspondence to the direction of the magnetosheath field. This correspondence, and any dependence of magnetopause character on the magnetic shear across the boundary, is much weaker for earlier local time. Thus, the characteristics of the boundary region on the flanks is much less dependent on magnetic shear, than it is in the sub-solar region (as found by Phan et al., 1994). That there is a tendency for TD character to increase is perhaps not unexpected on the flanks, since reconnection is likely to be predominant in the sub-solar region. The existence of such large amplitude, compressional signatures in the adjacent magnetosheath is less common, however. It possibly results from convected signatures along the magnetosheath flow, rather than from locally generated effects, in view of the existence of both a high level of draping and often low shear (such as would be consistent with the formation of a plasma depletion layer). Unfortunately, the lack of routinely available plasma data at this time precludes a check on the plasma beta values in the adjacent magnetosheath.

Two events, which corresponded to conjunctions with the Geotail spacecraft, were included to provide some confirmation of the conclusions on boundary orientation and location. In both cases the MVA normals and relative timing of the crossings between each spacecraft were consistent not only with their locations, but in comparison to the respective model magnetopause surfaces, determined for Equator-S. The timing information allowed crude estimates of boundary motion in the two cases, giving relatively low speeds of order $10 \mathrm{~km} / \mathrm{s}$. For the second event (11 February), both spacecraft remained in contact with the magnetopause for longer, producing more than one similar crossing, and there was some evidence of rippling on the boundary which, although pronounced at Geotail, decayed towards Equator-S.

All events were routinely compared to the magnetic field models, where any sensitivity to solar wind conditions was checked and for which magnetospheric activity was accounted. We therefore chose to compare only the earlier field models which are relatively insensitive to external parameters. The field models generally match the set of events more poorly with decreasing local time, resulting in a systematic deviation between the data, T87 and T89 models which cannot be explained by these results indicating a blunter magnetopause boundary on the flank. Typically, the T87 model fits the data no worse than the T89 model, during conditions of low to intermediate $K_{P}$ index near the magnetopause, but it is often better for passes located further into the flank region. Near the dawn-side flank, where the magnetic field configuration is likely to be influenced by both the equatorial ring current and tail current sheet, T87 also fits the observed crossing of the magnetic equator better 
(typically, then at high $P_{\text {ram }}$ ). We propose this may be a result of the form of the tail current sheet used in T89, which remains warped near the dawn-dusk terminator.

Acknowledgements. This work was supported by PPARC. The Equator-S mission was made possible through grant 500C94024 by the German Space Agency (DARA/DLR). MWD thanks Dr T. Woodward for helpful suggestions on the text.

\section{References}

Acuna, M., Ogilvie, K. W., Baker, D. N., Curtis, S. A., Fairfield, D. H., and Mish, W. H., The global Geospace program and its investigations, Space Sci. Rev., 71, 5, 1995.

Anderson, B. J., and Fuselier, S. A., Magnetic pulsations from 0.1 to $4.0 \mathrm{~Hz}$ and associated plasma properties in the Earth's subsolar magnetosheath and plasma depletion layer, J. Geophys. Res., 98, 1461-1479, 1993.

Berchem, J. and Russell, C. T., The thickness of the magnetopause current layer: ISEE 1 and 2 observations, J. Geophys. Res., 87, 2108-2114, 1982.

Bryant, D. A., Krimigis S. M., and Haerendel G., Outline of the active magnetospheric particle tracer explorers (AMPTE) mission, IEEE Trans. Geosci. Remote Sensing, GE-23, 177, 1985.

Dunlop, M. W., Balogh, A., Baumjohann, W., Haerendel, G., Fornacon, K.-H. and Georgescu, E., Dawn-side magnetopause observed by the Equator-S magnetic field experiment: Identification and survey of crossings, in press, J. Geophys. Res., 1999.

Farrugia, C. J., Dunlop, M. W., Geurts, F., Balogh, A., Southwood, D. J., Bryant, D. A., Neugebauer, M., and Etemadi, A., An interplanetary magnetic structure orientated at a large ( $\sim 80 \mathrm{deg})$ angle to the Parker spiral, Geophys. Res. Lett., 17, 1025-1028, 1990.

Fairfield, D. H., Shape and location of the magnetopause, in Physics of the Magnetopause, Eds. Song P., Sonnerup B.U.O., and Thompsen, M.F. AGU Geophysical Monograph 90, 115122, 1995 .

Formisano, V., Domingo, V., and Wenzel, K.-P., The three dimensional shape of the magnetopause, Planet. Space Sci., 27, 1137-1149, 1979a.

Formisano V., Orientation and shape of the Earth's bow shock in three dimensions, Planet. Space Sci., 27, 1151-1161, 1979b.

Fornacon, K.-H., Auster, H. U., Georgescu, E., Baumjohann, W., Glassmeier, K.-H., Haerendel, G., Rustenbach, J., and Dunlop, M. W., The magnetic field experiment onboard Equator-S and it's scientific possibilities, this issue, Ann. Geo., 1999.

Lucek, E., Dunlop, M. W., Balogh, A. Cargill, P., Baumjohann, W., Georgescu, E., Haerendel, G., and Fornacon, K.-H., Mirror mode structures observed in the dawn-side magnetosheath by Equator-S, in press, Geophys. Res. Lett., 1999a.

Lucek, E., Dunlop, M. W., Balogh, A. Cargill, P., Baumjohann, W., Georgescu, E., Haerendel, G., and Fornacon, K.-H., Identification of magnetosheath mirror modes in Equator-S magnetic field data, this issue, Ann. Geo., 1999b.
Petrinec, S. P., Song P., and Russell, C. T., Solar cycle variations in the size and shape of the magnetopause, J. Geophys. Res., 96, 7893, 1991.

Phan, T.-D. and Paschmann, G., The magnetosheath region adjacent to the dayside magnetopause, in Physics of the Magnetopause, Eds. Song, Sonnerup and Thompsen, $A G U$ Geophysical Monograph 90, 115-122, 1995.

Phan, T.-D., Paschmann, G., Baumjohann, W., Sckopke, N., and Lühr, H., The magnetosheath region adjacent to the dayside magnetopause: AMPTE/IRM observations, J. Geophys. Res., 99, 121-141, 1994.

Paschmann, G., Papamastorakis, I., Baumjohann, W., Sckopke, N., Carlson, C. W., Sonnerup B. U. Ö., and Lühr, H., ISEE observations of the magnetopause: reconnection and the energy balance, J. Geophys. Res., 90, 12 111-12 120, 1985.

Paschmann, G., Papamastorakis, I., Baumjohann, W., Sckopke, N., Carlson, C. W., Sonnerup B. U. O., and Lühr, H., The magnetopause for large magnetic shear: AMPTE/IRM observations, J. Geophys. Res., 91, 11 099-11 115, 1986.

Russell, C. T., The structure of the magnetopause, in Physics of the Magnetopause, Eds. Song, Sonnerup and Thompsen, $A G U$ Geophysical Monograph 90, 81-98, 1995a.

Russell, C. T., The Global Geospace Mission, Space Science Rev., Kluwer Academic Publishers, 1995b.

Russell, C. T., and Elphic, R. C., Initial ISEE magnetometer results: magnetopause observations, Space Sci. Rev., 22, 681715, 1978.

Russell, C. T., and Elphic, R. C., ISEE observations of flux transfer events at the dayside magnetopause, Geophys. Res. Lett., 6, 33 $36,1979$.

Sibeck, D. G., Lopez R. E., and Roelof, E. C., Solar wind control of the magnetopause shape location and motion, J. Geophys. Res., 96, 5489, 1991.

Sonnerup, B. U. O., and Cahill, L. J., Magnetopause structure and attitude from Explorer 12 observations, J. Geophys. Res., 72, 171-183, 1967.

Song, P., Russell C. T., and Thompsen, M. F., Slow mode transition in the frontside magnetosheath, J. Geophys. Res., 97, 8295$8305,1992 \mathrm{a}$.

Song, P., Russell C. T., and Thompsen, M. F., Waves in the inner magnetosheath: a case study, Geophys. Res. Lett., 19, 21912194, 1992b.

Southwood, D. J., and Kivelson, M. G., On the form of the flow in the magnetosheath, J. Geophys. Res., 97, 2873, 1992.

Southwood, D. J., and Kivelson, M. G., The formation of slow mode fronts in the magnetosheath, in Physics of the Magnetopause, Eds. Song, Sonnerup and Thompsen, AGU Geophysical Monograph 90, 109-114, 1995.

Spreiter, J. R., Summers, A. L., and Alksne, Y., Hydromagnetic flow around the magnetosphere, Planet. Space Sci., 14, 223-253, 1966.

Tsyganenko N. A., and Usmanov, A. V., Global quantitative models of the geomagnetic field in the cislunar magnetosphere for different disturbance levels, Planet Space Sci., 35, 11, 1347$1358,1987$.

Tsyganenko N. A., A magnetospheric field model with a warped tail current sheet, Planet. Space Sci., 37, 1, 5-20, 1989. 